Article

\title{
The Development and Validation of a Novel "Dual Cocktail" Probe for Cytochrome P450s and Transporter Functions to Evaluate Pharmacokinetic Drug-Drug and Herb-Drug Interactions
}

\author{
Mihwa Kwon ${ }^{1}$, Ji-Hyeon Jeon ${ }^{1}$, Min-Koo Choi ${ }^{2}$ and Im-Sook Song ${ }^{1,3, * \mathbb{D}}$ \\ 1 College of Pharmacy and Research Institute of Pharmaceutical Sciences, Kyungpook National University, \\ Daegu 41566, Korea; mihwa_k@naver.com (M.K.); kei7016@naver.com (J.-H.J.) \\ 2 College of Pharmacy, Dankook University, Cheon-an 31116, Korea; minkoochoi@dankook.ac.kr \\ 3 Vessel-Organ Interaction Research Center (VOICE), Kyungpook National University, Daegu 41566, Korea \\ * Correspondence: isssong@knu.ac.kr; Tel.: +82-53-950-8575
}

Received: 24 August 2020; Accepted: 29 September 2020; Published: 30 September 2020

\begin{abstract}
This study was designed to develop and validate a 10 probe drug cocktail named "Dual Cocktail", composed of caffeine (Cyp1a2 in rat and CYP1A2 in human, $1 \mathrm{mg} / \mathrm{kg}$ ), diclofenac (Cyp2c11 in rat and CYP2C9 in human, $2 \mathrm{mg} / \mathrm{kg}$ ), omeprazole (Cyp2c11 in rat and CYP2C19 in human, $2 \mathrm{mg} / \mathrm{kg}$ ), dextromethorphan (Cyp2d2 in rat and CYP2D6 in human, $10 \mathrm{mg} / \mathrm{kg}$ ), nifedipine (Суp3a1 in rat and CYP3A4 in human, $0.5 \mathrm{mg} / \mathrm{kg}$ ), metformin (Oct1/2 in rat and OCT1/2 in human, $0.5 \mathrm{mg} / \mathrm{kg}$ ), furosemide (Oat $1 / 3$ in rat and OAT1 $/ 3$ in human, $0.1 \mathrm{mg} / \mathrm{kg}$ ), valsartan (Oatp2 in rat and OATP1B1/1B3 in human, $0.2 \mathrm{mg} / \mathrm{kg}$ ), digoxin (P-gp in rat and human, $2 \mathrm{mg} / \mathrm{kg}$ ), and methotrexate (Mrp2 in rat and MRP2 in human, $0.5 \mathrm{mg} / \mathrm{kg}$ ), for the evaluation of pharmacokinetic drug-drug and herb-drug interactions through the modulation of a representative panel of CYP enzymes or transporters in rats. To ensure no interaction among the ten probe substrates, we developed a 2-step evaluation protocol. In the first step, the pharmacokinetic properties of five individual CYP probe substrates and five individual transporter substrates were compared with the pharmacokinetics of five CYP cocktail or five transporters cocktails in two groups of randomly assigned rats. Next, a pharmacokinetic comparison was conducted between the CYP or transporter cocktail group and the dual cocktail group, respectively. None of the ten comparison groups was found to be statistically significant, indicating the CYP and transporter substrate sets or dual cocktail set could be concomitantly administered in rats. The "Dual Cocktail" was further validated by assessing the metabolism of nifedipine and omeprazole, which was significantly reduced by a single oral dose of ketoconazole $(10 \mathrm{mg} / \mathrm{kg})$; however, no changes were observed in the pharmacokinetic parameters of other probe substrates. Additionally, multiple oral doses of rifampin $(20 \mathrm{mg} / \mathrm{kg})$ reduced the plasma concentrations of nifedipine and digoxin, although not any of the other substrates. In conclusion, the dual cocktail can be used to characterize potential pharmacokinetic drug-drug interactions by simultaneously monitoring the activity of multiple CYP isoforms and transporters.
\end{abstract}

Keywords: dual cocktail; pharmacokinetic drug-drug interaction; cytochrome P450 (CYP); transporter

\section{Introduction}

The evaluation of potential drug-drug interactions (DDIs) is becoming increasingly significant because of frequent concomitant use of multi-drugs among individuals over 60 years old, and the overuse of health supplements or over the counter drugs [1]. Owing to the growing use of herbal supplements, adverse drug reactions or herb-drug interactions (HDIs) resulting from the 
co-administration of therapeutic drugs with herbal supplements have also rapidly increased [2]. In China, herbal medicine formulations have accounted for approximately $13.8 \%$ and $17.3 \%$ of the total adverse drug reactions in 2010 and 2013, respectively [2]. The quantitative prediction of DDIs between co-administered therapeutic drugs using physiologically based pharmacokinetic modeling (PBPK) approaches has been widely used. However, PBPK approaches for HDIs between therapeutic drugs and herbal supplements have been limited to several herbs, such as St. John's wort and milk thistle [3,4]. These approaches were performed using hyperforin and silybin, the major and well-characterized component of St. John's wort and silymarin, respectively [3,4]. This limitation depends on the features of herbal medicines. Herbal supplements are bioactive constituent mixtures that substantially vary depending on the preparation methods [4]. Moreover, several bioactive constituents in one herbal supplement may coordinately interact with therapeutic drugs due to their similar inhibitory characteristics, although the pharmacokinetic features of these bioactive components have not been fully investigated [1,4]. In certain circumstances, such as when multiple components simultaneously inhibited the drug-metabolizing enzymes and transporters as well as when the pharmacokinetics of these components are not fully characterized, an in vivo cocktail approach may have advantages to efficiently evaluate potential DDIs or HDIs. The in vivo cocktail approach is a combination of several probe drugs that are sensitive to the modulation of specific metabolic enzymes or transporter proteins. This strategy is effective in characterizing the potential in vivo pharmacokinetic interactions between the probe drug and the potential perpetrator. Recent studies have focused on phenotyping major metabolizing enzymes in human and rat [5-8]. Among these cocktails "Inje cocktail [7]" which includes caffeine (cytochrome P450 [CYP] 1A2), omeprazole (CYP2C19), losartan (CYP2C9), dextromethorphan (CYP2D6), and midazolam (CYP3A), has been successfully used in rat cocktail studies [8]. In this study, significant DDIs in probe substrates were not detected even at high doses of caffeine $(1 \mathrm{mg} / \mathrm{kg})$, omeprazole $(40 \mathrm{mg} / \mathrm{kg})$, losartan $(10 \mathrm{mg} / \mathrm{kg})$, dextromethorphan $(10 \mathrm{mg} / \mathrm{kg})$, and midazolam $(10 \mathrm{mg} / \mathrm{kg}$ ) [8]. Videau et al. developed another cocktail set consisting of CYP enzyme and drug transporter modulators in both human and rat $[9,10]$. This cocktail set is composed of caffeine (CYP1A2), repaglinide (CYP2C8), tolbutamide (CYP2C9), omeprazole (CYP2C19), dextromethorphan (CYP2D6), midazolam (CYP3A), rosuvastatin (organic anion transporting polypeptides [OATPs]), acetaminophen (UGT), memantine (renal excretion), and digoxin (P-glycoprotein [P-gp]). The probe dose in rats is relatively low and within the range of $1-2 \mathrm{mg} / \mathrm{kg}$ [10]. Although this cocktail set included probes that are substrates for the OATPs and P-gp transporters, the use of transporter-targeted cocktails is still limited [11]. However, drug transporters are significant determinant of the pharmacokinetic features of drugs, and several clinically relevant DDIs or HDIs were mediated through drug transporter inhibition, as evidenced by numerous reports in the literature $[1,12,13]$. Therefore, this study aimed to develop a combination of dual cocktails that consists of five drugs as probes for CYP metabolizing enzyme function and five drugs as probes for transporter function to simultaneously monitor potential pharmacokinetic DDIs or HDIs in rats in vivo.

The probes were selected based on their safety profiles, ease of use, specificity, and the significance of their modifying metabolizing enzymes and transporters in pharmacokinetic DDIs according to the Food and Drug Administration (FDA) and European Medicines Agency (EMA) guidelines [14,15]. Notably, the lack of interaction among the cocktail substrates should have been demonstrated in vivo [15]. CYP1A2 (Cyp1a2 in rat), CYP2C9 (Cyp2c11 in rat), CYP2D6 (Cyp2d2 in rat), CYP2C19 (Cyp2c11 in rat), and CYP3A4 (Cyp3a1 in rat) were selected as the key drug metabolizing enzymes involved in DDIs and HDIs [7,8,16]. Caffeine, omeprazole, and dextromethorphan were selected as substrates for Cyp1a2, Cyp2c11, and Cyp2d2 based on the previous reports $[7,8]$. Nifedipine was selected as a substrate for Сyp3a1 based on previous results showing that the formation of dehydronifedipine from nifedipine is catalyzed by Cyp3a1 in rats, and nifedipine pharmacokinetics was modulated by the treatment of Cyp3a1 inhibitors [17,18]. Diclofenac was selected because Cyp2c11 is mainly involved in its metabolism [19]. The probes were used at the lowest dose that can reduce a DDI possibility among cocktail probe substrates according to the previously suggested in vivo 
animal cocktail (dose range, $0.5-2 \mathrm{mg} / \mathrm{kg}$ ) [10]. In the case of dextromethorphan, we selected its dose as $10 \mathrm{mg} / \mathrm{kg}$ to access its plasma profile based on the study of Uchida et al. [8]. Therefore, to develop CYP cocktail set, caffeine $(1 \mathrm{mg} / \mathrm{kg})$, diclofenac $(2 \mathrm{mg} / \mathrm{kg})$, dextromethorphan $(10 \mathrm{mg} / \mathrm{kg})$, omeprazole $(2 \mathrm{mg} / \mathrm{kg})$, and nifedipine $(0.5 \mathrm{mg} / \mathrm{kg})$ were selected as their probe substrates $[16,17,19]$.

$\mathrm{P}$-gp (P-gp in rat), organic anion transporters (OATs; Oats in rat), organic cation transporters (OCTs; Octs in rat), multidrug resistance-associated protein 2 (MRP2; Mrp2 in rat), and OATPs (Oatps in rat) were selected as the key drug transporters involved in DDIs [11]. Digoxin $(2 \mathrm{mg} / \mathrm{kg})$, furosemide $(0.1 \mathrm{mg} / \mathrm{kg})$, metformin $(0.5 \mathrm{mg} / \mathrm{kg})$, methotrexate $(0.5 \mathrm{mg} / \mathrm{kg})$, and valsartan $(0.2 \mathrm{mg} / \mathrm{kg})$ were selected as their probe substrates because Octs, Oats, P-gp, and Mrp2 play significant roles in the disposition and plasma profile of these substrate drugs [11,13,20,21]. These substrates were intravenously administered due to their low bioavailability, which is below 30\% [13,22-26]. The probe substrate doses were also selected as the lowest dose that could access full plasma profile in rat from the preliminary study. These substrates, which do not include any controlled substance, are typically used in both human and animals with optimal ease of access.

Here, we designed a two-step validation process to develop and validate the dual cocktail (Figure 1). In the first development phase, a cocktail of five CYP substrates and a cocktail of five transporter substrates combinations were separately validated to ensure no interactions exist among the selected substrates. In the second development phase, the cocktails of CYP substrates and transporter substrates were concurrently tested in rats to ensure that there are no interactions among these 10 probes. This dual cocktail was further validated with representative CYP or transporter inhibitors or inducers as well as red ginseng extract (RGE), a frequently used herbal supplement $[27,28]$, to investigate the potential pharmacokinetic DDIs or HDIs associated with representative inhibitors or herbal supplements in rats.

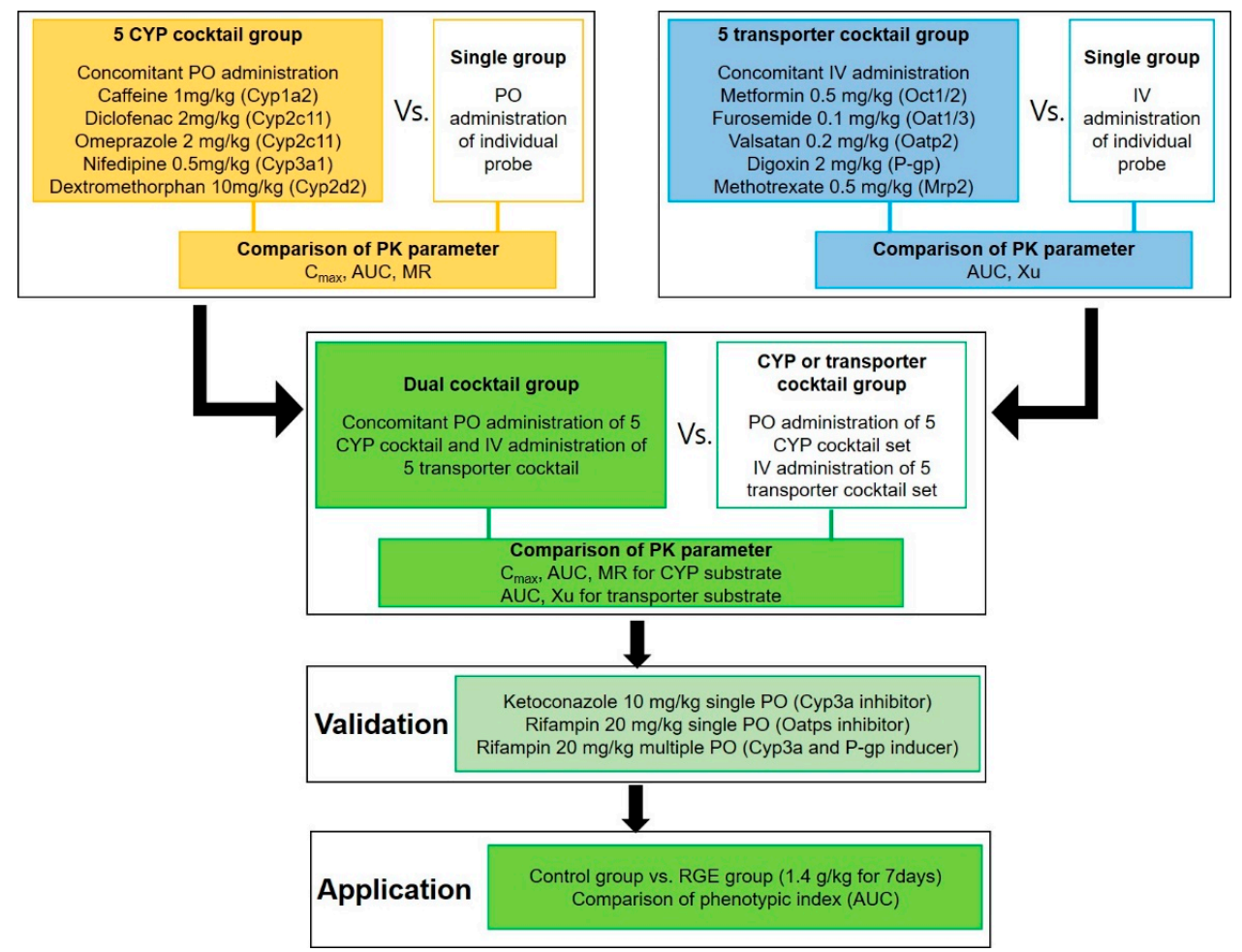

Figure 1. Strategy for the "Dual Cocktail" development. CYP; cytochrome P450, PO; per oral, IV; intravenous, $\mathrm{PK}$, Pharmacokinetics, $\mathrm{C}_{\mathrm{max}}$; maximum plasma concentration, AUC; area under plasma concentration curve, MR; metabolic ratio calculated by dividing metabolite AUC by parent $\mathrm{AUC}, \mathrm{Xu}$; excreted amount of substrate via renal route, RGE; red ginseng extract.

RGE is one of the most popular herbal medicines in several countries, including East Asia [1]. Discrepancies have been observed in the HDIs of RGE in both human and animals. Gurley et al. [29,30] 
reported no significant CYP-mediated HDIs in young and elderly healthy subjects from the measurement of CYP1A2, CYP2E1, CYP3A4, and CYP2D6 activities following the oral administration of $0.5 \mathrm{~g}$ Korean ginseng powder twice daily ( $75 \mathrm{mg}$ ginsenoside/day) for 28 days. In vivo cocktail approaches using a modified Inje cocktail to investigate HDIs between RGE (64\% dried ginseng, once daily for two weeks, 85-100 mg ginsenoside/day) and CYP probe substrates in healthy Koreans reported a weak interaction of RGE with CYP2C9, CYP2C19, CYP3A4, and CYP2D6, but no interaction with P-gp and OATP1B1 [31,32]. In a study conducted by Malati et al. [33], Korean ginseng ( $0.5 \mathrm{~g}$ capsule twice daily for 28 days, $50 \mathrm{mg}$ ginsenoside/day) induced CYP3A activity following the oral administration of $8 \mathrm{mg}$ midazolam. In animal study, a repeated oral administration of RGE $(0.5 \mathrm{~g} / \mathrm{kg}$ for 2 weeks; equivalent to $4.05 \mathrm{mg}$ ginsenoside $/ \mathrm{kg}$ ) did not alter the metabolic activity of Cyp1a, Сyp2b, Сyp2c, Cyp2d, and Сyp3a in the mouse liver [34]. However, another study revealed that the oral administration of the ethanol extract of ginseng ( $30 \mathrm{mg} / \mathrm{kg}$ for 10 days; equivalent to $8.13 \mathrm{mg}$ ginsenoside $/ \mathrm{kg}$ ) to rats increased the mRNA expression of Cyp2d2 and Cyp3a1 in the rat liver [35]. Although the mechanism was not unveiled, the discrepancy between the two studies can be explained from different ginsenoside concentrations and compositions of the ginseng product used. For the conclusive investigation on the pharmacokinetic HDI of RGE, we selected a high-RGE dose $(1.4 \mathrm{~g} / \mathrm{kg} /$ day for 1 week, $26.5 \mathrm{mg}$ ginsenosides/day) based on its therapeutic efficacy and investigated the modulatory effect of RGE on the 10 probe substrates for drug-metabolizing enzymes and transporters simultaneously using the developed "Dual Cocktail" set. RGE has been reported to be effective in various disease animal models, including diabetes, cancer, and arthritis, in the dose range of $50-2.0 \mathrm{~g} / \mathrm{kg}$ (containing 3-25 mg/kg of total ginsenosides or $20 \mathrm{mg} / \mathrm{kg}$ ginsenoside Rc) [36-41].

\section{Materials and Methods}

\subsection{Materials}

Caffeine, diclofenac, dextromethorphan, nifedipine, omeprazole, digoxin, furosemide, metformin, methotrexate, valsartan, and berberine were purchased from Sigma-Aldrich (St. Louis, MO, USA). Paraxanthine, 4-hydroxy diclofenac, dextrorphan, dehydronifedipine, and 5-hydroxy omeprazole were purchased from Toronto Research Chemicals Inc. (North York, ON, Canada). All other chemicals and solvents are of reagent and HPLC grades.

RGE was obtained from Punggi Ginseng Cooperative Association (Punggi, Korea). RGE contains $>60 \%$ of dried ginseng and total ginsenosides of $18.9 \mathrm{mg} / \mathrm{g}$. This product was produced in the facilities under the current guidelines of the Korea Good Manufacturing Practice (Lot No. 71921092). Individual ginsenoside content is provided in Table 1.

Table 1. Ginsenoside content in red ginseng extract (RGE) product.

\begin{tabular}{cccc}
\hline \multicolumn{2}{c}{ Ginsenosides } & Content (mg/g RGE) & Total (mg/g RGE) \\
\hline & Ginsenoside Rb1 & 4.7 & \\
& Ginsenoside Rb2 & 2.3 & 14.3 \\
Protopanaxadiol-type & Ginsenoside Rc & 2.5 & \\
& Ginsenoside Rd & 1.3 & \\
& Ginsenoside Rg3 & 3.5 & 4.6 \\
Protopanaxatriol-type & Ginsenoside Re & 1.3 & \\
& Ginsenoside Rg1 & 0.6 & \\
& Ginsenoside Rf & 1.1 & \\
& Ginsenoside Rh1 & 1.6 & \\
\hline
\end{tabular}

\subsection{Animals and Ethical Approval}

Male Sprague-Dawley rats aged 7-8 weeks (Samtako, Osan, Korea) were housed under a $12 \mathrm{~h}$ light/dark cycle in a room with controlled temperature $\left(24 \pm 2^{\circ} \mathrm{C}\right)$ and humidity $(55 \pm 5 \%)$. Animals were 
acclimatized for 1 week in an animal facility at Kyungpook National University. Food and water were provided ad libitum. All animal experiments were approved by Institutional Animal Care and Use Committee of Kyungpook National University (Approval No. KNU 2017-21, 17 February 2017).

\subsection{Pharmacokinetic Study}

For the development of 5 CYP cocktail (Figure 1), rats were randomly divided into the 5 CYP cocktail group ( $n=6$ ) and single groups ( $n=6$ each for individual probe substrate administration). The femoral arteries and femoral veins of rats were cannulated with PE50 polyethylene tubing (Jungdo, Seoul, Korea) under anesthesia with zoletil and lompun (50 and $5 \mathrm{mg} / \mathrm{kg}$, respectively, intramuscular injection) and heparinized saline $(10 \mathrm{U} / \mathrm{mL})$ was used to prevent blood clotting. Pharmacokinetic studies were initiated after the recovery from anesthesia. Each rat in the 5 CYP cocktail group received the 5 CYP cocktail solution $(2 \mathrm{~mL} / \mathrm{kg})$, including caffeine $(1 \mathrm{mg} / \mathrm{kg})$, diclofenac $(2 \mathrm{mg} / \mathrm{kg})$, dextromethorphan $(10 \mathrm{mg} / \mathrm{kg})$, omeprazole $(2 \mathrm{mg} / \mathrm{kg})$, and nifedipine $(0.5 \mathrm{mg} / \mathrm{kg})$. The probes were dissolved in a saline solution containing 10\% DMSO. The rats in the single groups received individual CYP probe substrate solution orally with the same dose and volume as the cocktail solution. Blood samples were collected via the femoral artery at $0,0.25,0.5,1,2,4,8$, and $24 \mathrm{~h}$ following the oral probe substrate administration, and a normal saline solution was administered via the femoral vein to compensate for blood sampling. After the centrifugation of blood samples at $8000 \times g$ for $1 \mathrm{~min}, 50 \mu \mathrm{L}$ aliquots of plasma samples were stored at $-80{ }^{\circ} \mathrm{C}$ until the analysis of probe substrates and their metabolites.

For the 5 transporter cocktail development (Figure 1), the rats were randomly divided into the 5 transporter cocktail $(n=6)$ and single groups $(n=6$ each) for individual probe substrate studies. The femoral arteries and veins of the rats were cannulated with PE50 polyethylene tubing (Jungdo, Seoul, Korea) under anesthesia with Zoletil and lompun (50 and $5 \mathrm{mg} / \mathrm{kg}$, respectively, intramuscular injection), and heparinized saline $(10 \mathrm{U} / \mathrm{mL})$ was used to prevent blood clotting. Pharmacokinetic studies were initiated after recovery from the anesthesia. Each rat in the 5 transporter cocktail groups received an intravenous cocktail $(1 \mathrm{~mL} / \mathrm{kg})$ of 5 solutions, which included digoxin $(2 \mathrm{mg} / \mathrm{kg})$, furosemide $(0.1 \mathrm{mg} / \mathrm{kg})$, metformin $(0.5 \mathrm{mg} / \mathrm{kg})$, methotrexate $(0.5 \mathrm{mg} / \mathrm{kg})$, and valsartan $(0.2 \mathrm{mg} / \mathrm{kg})$. The drugs were dissolved in saline solution containing $10 \% \mathrm{DMSO}$. The rats within the single group studies received individual substrate solutions intravenously with an equivalent dose and volume to the cocktail solution. Blood samples were collected via the femoral artery at $0,0.25,0.5$, $1,2,4,8$, and $24 \mathrm{~h}$ following the intravenous injection of probe substrates. Normal saline solutions were administered via the femoral vein to compensate for blood sampling. After the centrifugation of blood samples at $8000 \times g$ for $1 \mathrm{~min}, 50 \mu \mathrm{L}$ aliquots of plasma samples were stored at $-80{ }^{\circ} \mathrm{C}$ until the analysis of probe substrates.

For the dual cocktail development (Figure 1), the rats were randomly divided into three groups to receive the 5 CYP cocktail $(n=6), 5$ transporter cocktail $(n=6)$, and dual cocktail set $(n=7)$. The femoral arteries and veins of rats were cannulated with PE50 polyethylene tubing (Jungdo, Seoul, Korea) under anesthesia with Zoletil and lompun (50 and $5 \mathrm{mg} / \mathrm{kg}$, respectively, intramuscular injection), and heparinized saline $(10 \mathrm{U} / \mathrm{mL})$ was used to prevent blood clotting. Pharmacokinetic studies were started after the recovery from the anesthesia. The rats in the 5 CYP cocktail group received the 5 CYP substrate mixture orally. The rats in the 5 transporter cocktail group received the 5 transporter substrate mixture intravenously as previously described. The rats in the dual cocktail group simultaneously received the oral 5 CYP cocktail solution and the intravenous 5 transporter cocktail solution at an equivalent dose and similar method previously described. Blood samples were collected via the femoral artery at 0 , $0.25,0.5,1,2,4,8$, and $24 \mathrm{~h}$ following the probe substrate administration.

For the CYP3A inhibition (Figure 1), the rats were randomly divided into the control $(n=3)$ and single ketoconazole $(10 \mathrm{mg} / \mathrm{kg})$ groups $(n=4)$. The rats in the ketoconazole group received ketoconazole solution $(10 \mathrm{mg} / \mathrm{mL} / \mathrm{kg})$ via oral gavage, while the control rats received vehicle $(1 \mathrm{~mL} / \mathrm{kg})$ also via oral gavage. After $1 \mathrm{~h}$ of ketoconazole treatment, the dual cocktail mixture solution was administered as previously described. Blood samples were collected via the femoral artery at $0,0.25,0.5,1,2,4,8$, 
and $24 \mathrm{~h}$ following the probe substrate administration. For the OATPs inhibition (Figure 1 ), the rats were randomly divided into the control $(n=3)$ and single rifampin $(20 \mathrm{mg} / \mathrm{kg})$ groups $(n=4)$. The rats in the rifampin group received rifampin solution $(20 \mathrm{mg} / \mathrm{mL} / \mathrm{kg})$ via oral gavage, while the control rats received vehicle $(1 \mathrm{~mL} / \mathrm{kg})$ also via oral gavage. After $1 \mathrm{~h}$ of rifampin treatment, the dual cocktail mixture was administered as previously described. Blood samples were collected via the femoral artery at $0,0.25,0.5,1,2,4,8$, and $24 \mathrm{~h}$ following the probe substrate administration. For the induction study (Figure 1), the rats were randomly divided into the control $(n=4)$ and multiple rifampin $(20 \mathrm{mg} / \mathrm{kg} /$ day for 5 days) groups $(n=4)$. The rats in the rifampin group received rifampin solution $(20 \mathrm{mg} / \mathrm{mL} / \mathrm{kg})$ via oral gavage, while the control rats received vehicle ( $1 \mathrm{~mL} / \mathrm{kg}$ for 5 days) also via oral gavage. After $24 \mathrm{~h}$ of the last rifampin treatment, the dual cocktail mixture was administered as previously described to avoid the direct inhibitory effect of rifampin on drug transporters [42]. Blood samples were collected via the femoral artery at $0,0.25,0.5,1,2,4,8$, and $24 \mathrm{~h}$ following the oral probe substrate administration. After the centrifugation of blood samples at $8000 \times g$ for $1 \mathrm{~min}, 50 \mu \mathrm{L}$ aliquots of plasma samples were stored at $-80^{\circ} \mathrm{C}$ until the analysis of probe substrates and their metabolites.

For the dual cocktail application (Figure 1), the rats were randomly divided into the control $(n=7)$ and multiple RGE treatment $(1.4 \mathrm{~g} / \mathrm{kg}$ for 7 days) groups $(n=7)$. The rats in the multiple RGE group received RGE suspension $(1.4 \mathrm{~g} / \mathrm{mL} / \mathrm{kg} /$ day) for 7 days orally via oral gavage. The control group received water $(1 \mathrm{~mL} / \mathrm{kg})$ for 7 days by oral gavage. After $1 \mathrm{~h}$ of the last RGE treatment, the rats received the oral 5 CYP cocktail solution and simultaneously received the intravenous 5 transporter cocktail mixture at the same dose and method previously described. Blood samples were collected via the femoral artery at $0,0.25,0.5,1,2,4,8$, and $24 \mathrm{~h}$ following the probe substrate administration. After the centrifugation of blood samples at $8000 \times g$ for $1 \mathrm{~min}, 50 \mu \mathrm{L}$ aliquots of plasma samples were stored at $-80{ }^{\circ} \mathrm{C}$ until the analysis of probe substrates and their metabolites.

The samples were prepared using protein precipitation. The samples $(50 \mu \mathrm{L})$ were then added to $200 \mu \mathrm{L}$ of internal standard (IS) working solution (berberine $2 \mathrm{ng} / \mathrm{mL}$ in methanol). After vortex mixing of the sample mixture for $15 \mathrm{~min}$ and centrifugation at $16,000 \times g$ for $5 \mathrm{~min}, 5 \mu \mathrm{L}$ of the supernatant was injected into the LC-MS/MS system.

\subsection{LC-MS/MS Analysis of Probe Substrate}

The concentrations of 15 probe substrates and metabolites for CYP isoforms and transporters were simultaneously analyzed using an Agilent 6470 Triple Quadrupole LC-MS/MS system (Agilent, Wilmington, DE, USA). The ionization mode and mass transition for Q1 to Q3 were selected based on the product ion scan results of authentic standards and previously published reports [13,43-49]. The selected probe substrates and metabolites for CYP isoforms and transporters and their mass conditions are summarized in Table 2, including the ionization mode, mass transition, and collision energy. All analytes, with the exception of furosemide, were analyzed in the positive ionization mode.

In addition, the separation of 15 analytes and IS was performed on a Polar RP column $(2.0 \times 150 \mathrm{~mm}$, $5 \mu \mathrm{m}$; Phenomenex, Torrance, CA, USA) using a mobile phase consisting of water: acetonitrile $(30: 70 \mathrm{v} / \mathrm{v})$ with $0.1 \%$ formic acid at a $0.2 \mathrm{~mL} / \mathrm{min}$ flow rate. The retention time and the linear range of the standard curve mixture of all analytes are also shown in Table 2.

The intra- and inter-day precision and accuracy were analyzed for the six replicates at three QC samples $(5,50$, and $250 \mathrm{ng} / \mathrm{mL})$ for all analytes. Short-term stability was assessed by placing QC samples at $25^{\circ} \mathrm{C}$ for $4 \mathrm{~h}$. The stability of three freeze-thaw cycles was analyzed by comparing the QC samples ( 5 and $250 \mathrm{ng} / \mathrm{mL}$ ) that underwent three freeze-thaw cycles (from $-80{ }^{\circ} \mathrm{C}$ to $25^{\circ} \mathrm{C}$ for $4 \mathrm{~h}$ as one cycle) with those of the control group. Post-treatment stability was evaluated by placing the processed QC samples in the autosampler at $6^{\circ} \mathrm{C}$ for $24 \mathrm{~h}$.

\subsection{Data Analysis}

The pharmacokinetic parameters were calculated based on the plasma concentration-time profile using a non-compartment analysis by WinNonlin (version 5.1; Pharsights, Cary, NC, USA). 
Table 2. MS/MS parameters for the detection of 15 probe substrates and metabolites.

\begin{tabular}{|c|c|c|c|c|c|}
\hline Analyte & Mode & $m / z \mathrm{Q} 1 \rightarrow \mathrm{Q} 3$ & Collision Energy (V) & Retention Time (min) & Linear Range $(\mathrm{ng} / \mathrm{mL})$ \\
\hline Caffeine & positive & $194.9 \rightarrow 138.0$ & 20 & 4.09 & $1-1000$ \\
\hline Paraxanthine & positive & $180.7 \rightarrow 124.3$ & 10 & 2.97 & $1-1000$ \\
\hline Diclofenac & positive & $295.9 \rightarrow 215.0$ & 20 & 9.58 & $1-1000$ \\
\hline 4-Hydroxydiclofenac & positive & $312.2 \rightarrow 230.9$ & 20 & 5.25 & $1-1000$ \\
\hline Dextromethorphan & positive & $272.0 \rightarrow 214.9$ & 25 & 3.37 & $1-1000$ \\
\hline Dextrorphan & positive & $258.0 \rightarrow 201.0$ & 25 & 2.42 & $1-1000$ \\
\hline Nifedipine & positive & $347.0 \rightarrow 315.0$ & 5 & 6.16 & $1-1000$ \\
\hline Dehydronifedipine & positive & $245.0 \rightarrow 283.9$ & 30 & 6.44 & $1-1000$ \\
\hline Omeprazole & positive & $346.0 \rightarrow 197.8$ & 5 & 4.29 & $1-1000$ \\
\hline 5-Hydroxyomeprazole & positive & $362.2 \rightarrow 213.9$ & 10 & 3.26 & $1-1000$ \\
\hline Digoxin & positive & $803.4 \rightarrow 283.0$ & 50 & 4.35 & $5-2000$ \\
\hline Furosemide & negative & $328.9 \rightarrow 284.8$ & 15 & 3.62 & $1-2000$ \\
\hline Metformin & positive & $129.9 \rightarrow 60.0$ & 10 & 2.05 & $1-2000$ \\
\hline Methotrexate & positive & $455.1 \rightarrow 308.2$ & 20 & 2.23 & $1-2000$ \\
\hline Valsartan & positive & $436.1 \rightarrow 291.0$ & 5 & 7.22 & $1-2000$ \\
\hline Berberine & positive & $336.1 \rightarrow 320.0$ & 30 & 5.07 & \\
\hline
\end{tabular}

The statistical significance was assessed by a non-parametric Mann-Whitney test using the Statistical Package for the Social Sciences (version 24.0; SPSS Inc., Chicago, IL, USA). Differences between groups were considered to be significant when the $p$-values were under 0.05 .

\section{Results}

\subsection{Simultaneous Determination of Selected Probe Substrate for CYP Enzymes and Transporters}

For the dual cocktail set development in rats, we applied a simultaneous determination of 15 substrates and metabolites in the rat plasma using an LC-MS/MS system based on the product ion scan results of authentic standards and previously published reports [13,43-49]. Representative multiple reaction-monitoring chromatograms for the selected probe substrates and metabolites of CYP enzymes and substrates for the transporters (Figure 2) showed that all the 15 probe substrates and metabolites were well separated with no interfering peaks at their respective retention times, suggesting that the 15 probe substrates and metabolites were simultaneously quantified in the rat plasma samples following the concomitant administration of 10 probe substrates. The lower limit of quantitation of the analytes were determined as $1 \mathrm{ng} / \mathrm{mL}$ for the 14 probe substrates or metabolites except for digoxin and $5 \mathrm{ng} / \mathrm{mL}$ for digoxin based on signal-to-noise ratios of over 10.0 [50]. The calibration curves showed good linearity over the range of $1-1000 \mathrm{ng} / \mathrm{mL}$ for CYP substrates and 1-2000 ng/mL for metformin, furosemide, valsartan, and methotrexate. The calibration curves of digoxin were linear in the concentration range of $5-2000 \mathrm{ng} / \mathrm{mL}$ (Table 2).

The precision and accuracy of intra- and inter-day assays are assessed using three different QC samples $(5,50,250 \mathrm{ng} / \mathrm{mL}$ of each probe substrate) and results are shown in Table 3 . In all cases, the intra- and inter-day precision and accuracy of the 15 analytes, including 10 probe substrates and five metabolites, are within $15 \%$ in the three QC sample concentrations $(5,50$, and $250 \mathrm{ng} / \mathrm{mL})$.

All analytes in plasma were stable for up to $4 \mathrm{~h}$ at $25{ }^{\circ} \mathrm{C}$ within $15 \%$ of standard deviation (SD) (Table 4). No significant degradation occurred in the low- and high-concentration QC samples of all analytes from the three freeze-thaw cycle stability and $24 \mathrm{~h}$ post-treatment stability measurement (Table 4). The results suggested that the analytical method developed using the LC-MS/MS system was reliable, reproducible, and accurate for the simultaneous analysis of the 10 CYP or transporter probe substrates and five CYP metabolites in rat plasma and, therefore, could be used for the development, validation, and application of the dual cocktail in rats. 


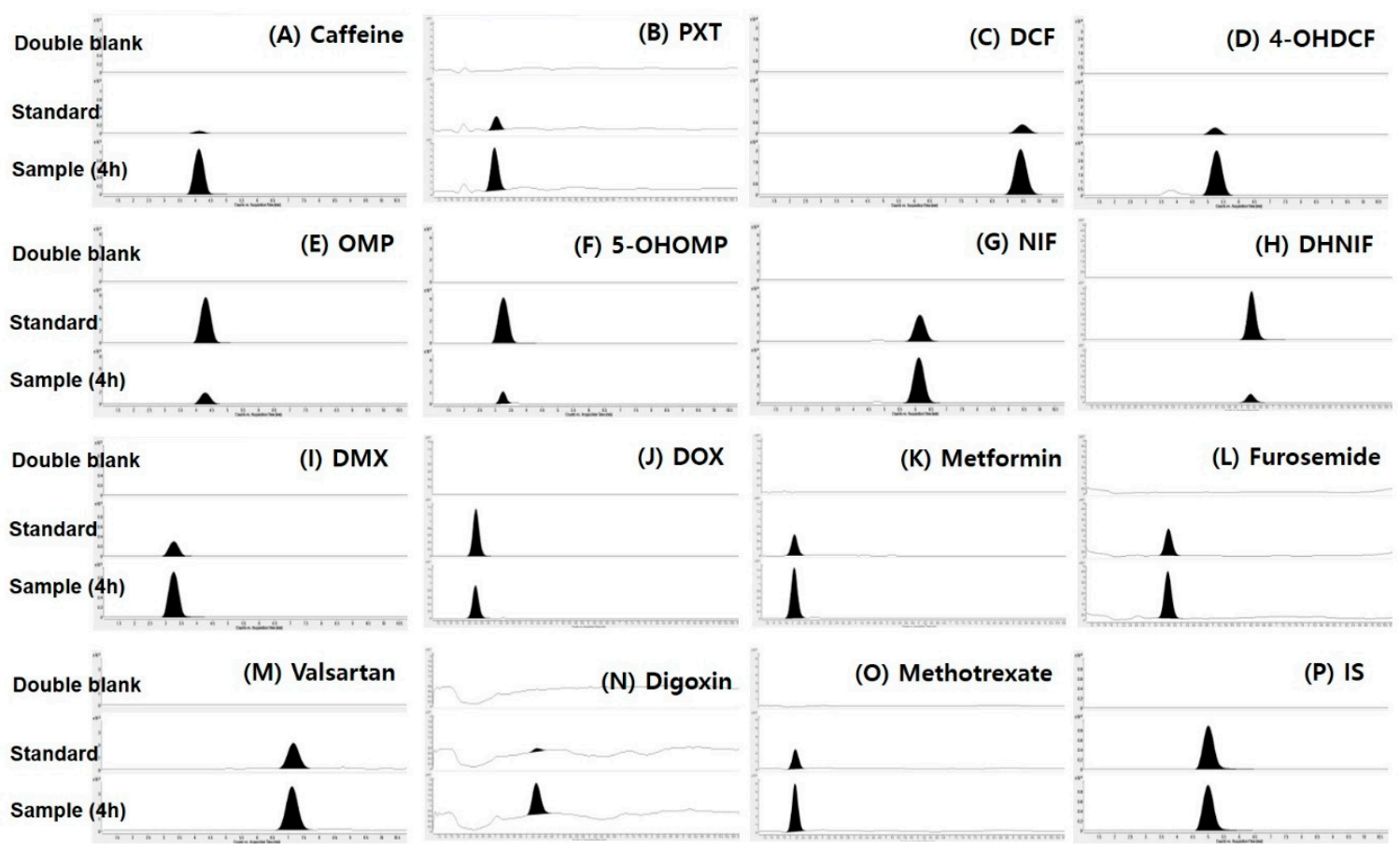

Figure 2. Representative multiple reaction-monitoring (MRM) chromatograms of the the selected probe substrates and metabolites for Cytochrome P450 (CYP) enzymes and substrates for transporters in rat double blank plasma (upper panel), rat blank plasma is spiked with a standard solution $(5 \mathrm{ng} / \mathrm{mL})$ of 15 probe substrates or metabolites to rats (middle panel), and rat plasma samples at $4 \mathrm{~h}$ following dual cocktail co-administration (lower panel). (A) Caffeine, (B) paraxanthine (PXT), (C) diclofenac (DCF), (D) 4-hydroxy diclofenac (4-OHDCF), (E) omeprazole (OMP), (F) 5-hydroxyomeprazole (5-OHOMP), (G) nifedipine (NIF), (H) dehydronifedipine (DHNIF), (I) dextromethorphan (DMX), (J) dextrorphan (DOX), (K) metformin, (L) furosemide, (M) valsartan, (N) digoxin, (O) methotrexate, and $(\mathbf{P})$ berberine (IS).

\subsection{Comparison of Five CYP Probe Substrate Simultaneously versus Individually Administered}

In the first step of five CYP cocktail development, we compared the plasma concentrations and the pharmacokinetic parameters of five CYP probe substrates between the cocktail and single administration groups. Figure $3 \mathrm{~A}$ shows the plasma concentrations of caffeine and its metabolite, paraxanthine, in the single and CYP cocktail groups, respectively. The $C_{\max }$ and AUC values of caffeine were similar in the single and CYP cocktail groups. The metabolic ratio (MR) calculated from the AUC of paraxanthine to the AUC of caffeine were similar in the single and CYP cocktail groups (Table 5), suggesting remote interactions in the pharmacokinetics and metabolic activity of caffeine and other probe substrates. Similarly, the plasma concentrations of diclofenac and 4-hydroxy diclofenac (Figure 3B), omeprazole and 5-hydroxyomeprazole (Figure 3C), nifedipine and dehydronifedipine (Figure 3D), and dextromethorphan and dextrorphan (Figure 3E) and their pharmacokinetic parameters (Table 5) were compared between the control and CYP cocktail groups. Table 3 reveals that the pharmacokinetic parameters of the probe substrates (i.e., diclofenac, omeprazole, nifedipine, and dextromethorphan) and their metabolites in the CYP cocktail group were not statistically different in the single administration group: $p>0.05$ for all parameters according to the Mann-Whitney $\mathrm{U}$ test. Collectively, these results suggested that 5 CYP cocktail substrates could be concomitantly administered to rats for the drug metabolic phenotype evaluation. The CYP cocktail included caffeine $(1 \mathrm{mg} / \mathrm{kg})$, diclofenac $(2 \mathrm{mg} / \mathrm{kg})$, omeprazole $(2 \mathrm{mg} / \mathrm{kg})$, nifedipine $(0.5 \mathrm{mg} / \mathrm{kg})$, and dextromethorphan $(10 \mathrm{mg} / \mathrm{kg})$ and was orally administered. 
Table 3. Intra- and inter-day precision and accuracy of 15 probe substrates and metabolites.

\begin{tabular}{|c|c|c|c|c|c|c|c|}
\hline \multirow{2}{*}{ Analyte } & \multirow{2}{*}{$\begin{array}{c}\text { Spiked Concentration } \\
(\mathrm{ng} / \mathrm{mL})\end{array}$} & \multicolumn{3}{|c|}{ Intra-Day } & \multicolumn{3}{|c|}{ Inter-Day } \\
\hline & & Measured (ng/mL) & Precision (\%) & Accuracy (\%) & Measured (ng/mL) & Precision (\%) & Accuracy (\%) \\
\hline \multirow{3}{*}{ Caffeine } & 5 & $4.65 \pm 0.41$ & 8.78 & 93.07 & $4.78 \pm 0.41$ & 8.66 & 95.51 \\
\hline & 50 & $46.42 \pm 1.94$ & 4.18 & 92.84 & $48.31 \pm 5.70$ & 11.81 & 96.63 \\
\hline & 250 & $253.58 \pm 11.79$ & 4.65 & 101.43 & $273.24 \pm 24.11$ & 8.83 & 109.30 \\
\hline \multirow{3}{*}{ Paraxanthine } & 5 & $5.17 \pm 0.40$ & 7.76 & 103.44 & $5.17 \pm 0.40$ & 7.76 & 103.44 \\
\hline & 50 & $43.66 \pm 1.89$ & 4.33 & 87.32 & $50.59 \pm 6.72$ & 13.28 & 101.18 \\
\hline & 250 & $222.27 \pm 2.77$ & 1.25 & 88.91 & $270.32 \pm 33.37$ & 12.34 & 108.13 \\
\hline \multirow{3}{*}{ Diclofenac } & 5 & $5.05 \pm 0.42$ & 8.35 & 100.90 & $5.17 \pm 0.55$ & 10.73 & 103.34 \\
\hline & 50 & $47.90 \pm 0.38$ & 0.79 & 95.80 & $47.89 \pm 5.28$ & 11.02 & 95.77 \\
\hline & 250 & $248.34 \pm 20.03$ & 8.07 & 99.34 & $244.29 \pm 16.02$ & 6.56 & 97.72 \\
\hline \multirow{3}{*}{ 4-Hydroxy diclofenac } & 5 & $4.54 \pm 0.31$ & 6.91 & 90.76 & $4.68 \pm 0.43$ & 9.24 & 93.51 \\
\hline & 50 & $49.19 \pm 1.59$ & 3.23 & 98.38 & $47.95 \pm 5.19$ & 10.83 & 95.90 \\
\hline & 250 & $261.64 \pm 18.42$ & 7.04 & 104.66 & $271.16 \pm 24.18$ & 8.92 & 108.47 \\
\hline \multirow{3}{*}{ Omeprazole } & 5 & $4.46 \pm 0.07$ & 1.65 & 89.27 & $5.33 \pm 0.65$ & 12.15 & 106.60 \\
\hline & 50 & $52.39 \pm 1.04$ & 1.99 & 104.79 & $53.48 \pm 4.79$ & 8.96 & 106.96 \\
\hline & 250 & $270.27 \pm 3.83$ & 1.42 & 108.11 & $276.90 \pm 18.04$ & 6.52 & 110.76 \\
\hline \multirow{3}{*}{ 5-Hydroxy omeprazole } & 5 & $4.53 \pm 0.10$ & 2.11 & 90.61 & $5.27 \pm 0.59$ & 11.12 & 105.32 \\
\hline & 50 & $51.48 \pm 2.96$ & 5.76 & 102.96 & $52.29 \pm 4.44$ & 8.49 & 104.57 \\
\hline & 250 & $274.62 \pm 2.60$ & 0.95 & 109.85 & $269.70 \pm 21.81$ & 8.09 & 107.88 \\
\hline \multirow{3}{*}{ Nifedipine } & 5 & $4.52 \pm 0.31$ & 6.89 & 90.38 & $4.43 \pm 0.36$ & 8.04 & 88.59 \\
\hline & 50 & $50.39 \pm 0.66$ & 1.31 & 100.79 & $46.45 \pm 5.28$ & 11.37 & 92.89 \\
\hline & 250 & $262.14 \pm 22.27$ & 8.49 & 104.85 & $254.12 \pm 21.95$ & 8.64 & 101.65 \\
\hline \multirow{3}{*}{ Dehydronifedipine } & 5 & $4.52 \pm 0.40$ & 8.94 & 90.32 & $4.62 \pm 0.39$ & 8.48 & 92.45 \\
\hline & 50 & $48.02 \pm 2.79$ & 5.81 & 96.04 & $50.67 \pm 1.28$ & 2.52 & 101.33 \\
\hline & 250 & $239.74 \pm 11.09$ & 4.63 & 95.90 & $272.04 \pm 18.83$ & 6.92 & 108.81 \\
\hline \multirow{3}{*}{ Dextromethorphan } & 5 & $4.50 \pm 0.22$ & 4.90 & 90.02 & $4.77 \pm 0.33$ & 6.91 & 95.46 \\
\hline & 50 & $50.88 \pm 1.25$ & 2.47 & 101.76 & $49.75 \pm 4.22$ & 8.47 & 99.50 \\
\hline & 250 & $269.65 \pm 11.78$ & 4.37 & 107.86 & $266.69 \pm 20.50$ & 7.69 & 106.68 \\
\hline \multirow{3}{*}{ Dextrorphan } & 5 & $4.43 \pm 0.04$ & 0.94 & 88.59 & $5.17 \pm 0.54$ & 10.36 & 103.43 \\
\hline & 50 & $52.82 \pm 5.04$ & 9.53 & 105.65 & $52.13 \pm 5.20$ & 9.97 & 104.27 \\
\hline & 250 & $276.68 \pm 6.37$ & 2.30 & 110.67 & $278.23 \pm 28.29$ & 10.17 & 111.29 \\
\hline \multirow{3}{*}{ Metformin } & 5 & $4.98 \pm 0.19$ & 3.82 & 99.59 & $5.25 \pm 0.28$ & 5.38 & 105.09 \\
\hline & 50 & $51.78 \pm 2.29$ & 4.43 & 103.55 & $49.67 \pm 5.39$ & 10.85 & 99.33 \\
\hline & 250 & $256.67 \pm 11.28$ & 4.39 & 102.67 & $235.72 \pm 7.66$ & 3.25 & 94.29 \\
\hline
\end{tabular}


Table 3. Cont

\begin{tabular}{|c|c|c|c|c|c|c|c|}
\hline \multirow{2}{*}{ Analyte } & \multirow{2}{*}{$\begin{array}{l}\text { Spiked Concentration } \\
(\mathrm{ng} / \mathrm{mL})\end{array}$} & \multicolumn{3}{|c|}{ Intra-Day } & \multicolumn{3}{|c|}{ Inter-Day } \\
\hline & & Measured (ng/mL) & Precision (\%) & Accuracy (\%) & Measured (ng/mL) & Precision (\%) & Accuracy (\%) \\
\hline \multirow{3}{*}{ Furosemide } & 5 & $4.77 \pm 0.46$ & 9.55 & 95.43 & $4.59 \pm 0.48$ & 10.47 & 91.89 \\
\hline & 50 & $45.98 \pm 2.29$ & 4.99 & 91.97 & $44.47 \pm 2.55$ & 5.74 & 88.95 \\
\hline & 250 & $247.19 \pm 9.61$ & 3.89 & 98.88 & $242.15 \pm 8.51$ & 3.51 & 96.86 \\
\hline \multirow{3}{*}{ Valsartan } & 5 & $4.85 \pm 0.45$ & 9.23 & 97.03 & $4.87 \pm 0.46$ & 9.48 & 97.31 \\
\hline & 50 & $49.07 \pm 0.88$ & 1.80 & 98.14 & $46.68 \pm 4.90$ & 10.51 & 93.35 \\
\hline & 250 & $270.50 \pm 5.55$ & 2.05 & 108.20 & $249.08 \pm 20.98$ & 8.42 & 99.63 \\
\hline \multirow{3}{*}{ Digoxin } & 5 & $4.95 \pm 0.06$ & 1.20 & 99.00 & $4.98 \pm 0.03$ & 0.59 & 99.65 \\
\hline & 50 & $44.16 \pm 2.58$ & 5.83 & 88.32 & $45.15 \pm 2.73$ & 6.06 & 90.29 \\
\hline & 250 & $245.12 \pm 13.38$ & 5.46 & 98.05 & $248.52 \pm 22.38$ & 9.01 & 99.41 \\
\hline \multirow{3}{*}{ Methotrexate } & 5 & $5.00 \pm 0.57$ & 11.38 & 100.05 & $5.03 \pm 0.57$ & 11.39 & 100.59 \\
\hline & 50 & $52.90 \pm 4.62$ & 8.74 & 105.80 & $45.11 \pm 5.41$ & 11.99 & 90.22 \\
\hline & 250 & $270.17 \pm 9.54$ & 3.53 & 108.07 & $251.27 \pm 21.74$ & 8.65 & 100.51 \\
\hline
\end{tabular}

The data expressed as the mean \pm standard deviation $(n=6)$. 
Table 4. Short-term, freeze-thaw cycle, and post-treatment stability of 15 probe substrates and metabolites.

\begin{tabular}{|c|c|c|c|c|}
\hline Analyte & $\begin{array}{l}\text { Spiked Concentration } \\
(\mathrm{ng} / \mathrm{mL})\end{array}$ & $\begin{array}{l}\text { Short Term Stability } \\
\left(4 \mathrm{~h}, 25^{\circ} \mathrm{C}\right)\end{array}$ & $\begin{array}{l}\text { Free-Thaw Cycle Stability } \\
\left(-80^{\circ} \mathrm{C} / 25^{\circ} \mathrm{C}, 3 \text { Cycles }\right)\end{array}$ & $\begin{array}{l}\text { Post Treatment Stability } \\
\qquad\left(6^{\circ} \mathrm{C}, 24 \mathrm{~h}\right)\end{array}$ \\
\hline \multirow{2}{*}{ Caffeine } & 5 & $96.9 \pm 7.9$ & $91.7 \pm 10.7$ & $92.4 \pm 12.9$ \\
\hline & 250 & $105.3 \pm 8.2$ & $108.5 \pm 7.7$ & $109.2 \pm 12.4$ \\
\hline \multirow{2}{*}{ Paraxanthine } & 5 & $90.7 \pm 9.8$ & $80.6 \pm 21.9$ & $82.0 \pm 15.7$ \\
\hline & 250 & $95.6 \pm 3.9$ & $107.8 \pm 7.4$ & $112.0 \pm 10.5$ \\
\hline \multirow{2}{*}{ Diclofenac } & 5 & $102.6 \pm 10.5$ & $108.3 \pm 21.4$ & $92.2 \pm 14.6$ \\
\hline & 250 & $106.6 \pm 10.7$ & $102.3 \pm 2.6$ & $103.2 \pm 3.3$ \\
\hline \multirow{2}{*}{ 4-Hydroxy diclofenac } & 5 & $98.1 \pm 6.5$ & $107.2 \pm 26.2$ & $91.6 \pm 12.9$ \\
\hline & 250 & $100.4 \pm 0.5$ & $94.7 \pm 5.6$ & $108.8 \pm 11.9$ \\
\hline \multirow{2}{*}{ Omeprazole } & 5 & $101.3 \pm 5.4$ & $100.2 \pm 17.8$ & $96.5 \pm 18.3$ \\
\hline & 250 & $105.0 \pm 8.7$ & $100.9 \pm 4.9$ & $109.4 \pm 10.5$ \\
\hline \multirow{2}{*}{ 5-Hydroxy omeprazole } & 5 & $103.1 \pm 3.1$ & $101.8 \pm 17.0$ & $98.3 \pm 7.4$ \\
\hline & 250 & $99.6 \pm 2.8$ & $104.6 \pm 4.0$ & $111.2 \pm 9.8$ \\
\hline \multirow{2}{*}{ Nifedipine } & 5 & $98.0 \pm 1.8$ & $94.4 \pm 10.8$ & $98.6 \pm 7.3$ \\
\hline & 250 & $99.7 \pm 1.9$ & $109.5 \pm 19.4$ & $106.5 \pm 16.3$ \\
\hline \multirow{2}{*}{ Dehydronifedipine } & 5 & $96.2 \pm 5.6$ & $86.9 \pm 12.5$ & $91.6 \pm 14.3$ \\
\hline & 250 & $103.1 \pm 6.4$ & $107.5 \pm 14.9$ & $111.8 \pm 10.9$ \\
\hline \multirow{2}{*}{ Dextromethorphan } & 5 & $94.8 \pm 4.7$ & $97.3 \pm 9.0$ & $91.1 \pm 11.3$ \\
\hline & 250 & $101.7 \pm 2.4$ & $101.5 \pm 4.0$ & $114.5 \pm 12.9$ \\
\hline \multirow{2}{*}{ Dextrorphan } & 5 & $105.1 \pm 5.7$ & $98.9 \pm 9.3$ & $98.1 \pm 10.2$ \\
\hline & 250 & $110.3 \pm 9.7$ & $96.6 \pm 3.7$ & $99.5 \pm 4.5$ \\
\hline \multirow{2}{*}{ Metformin } & 5 & $96.4 \pm 18.2$ & $104.8 \pm 4.5$ & $100.0 \pm 3.7$ \\
\hline & 250 & $102.7 \pm 6.8$ & $106.5 \pm 15.5$ & $102.9 \pm 14.2$ \\
\hline \multirow{2}{*}{ Furosemide } & 5 & $98.4 \pm 2.1$ & $90.1 \pm 9.2$ & $89.7 \pm 8.9$ \\
\hline & 250 & $101.3 \pm 1.2$ & $107.4 \pm 18.4$ & $105.5 \pm 14.2$ \\
\hline \multirow{2}{*}{ Valsartan } & 5 & $91.6 \pm 14.3$ & $101.5 \pm 1.3$ & $104.5 \pm 5.1$ \\
\hline & 250 & $102.8 \pm 3.5$ & $103.9 \pm 20.1$ & $101.2 \pm 12.9$ \\
\hline \multirow{2}{*}{ Digoxin } & 5 & $97.9 \pm 3.2$ & $89.6 \pm 9.2$ & $99.1 \pm 21.0$ \\
\hline & 250 & $96.9 \pm 3.8$ & $102.6 \pm 16.4$ & $97.8 \pm 7.0$ \\
\hline \multirow{2}{*}{ Methotrexate } & 5 & $96.6 \pm 4.1$ & $98.1 \pm 19.4$ & $92.6 \pm 6.4$ \\
\hline & 250 & $102.7 \pm 4.6$ & $106.4 \pm 21.3$ & $109.7 \pm 18.1$ \\
\hline
\end{tabular}

The data expressed as the mean \pm standard deviation $(n=3)$.
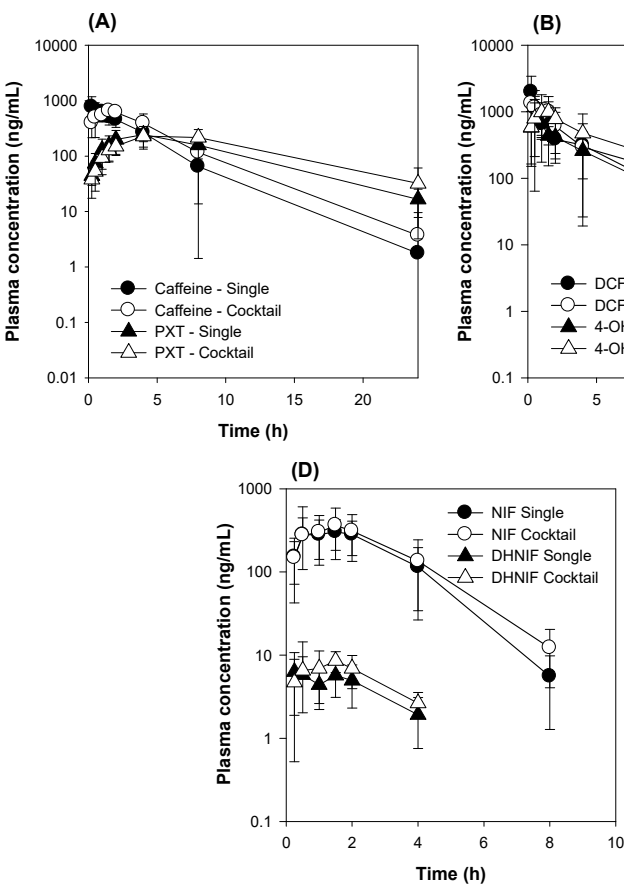

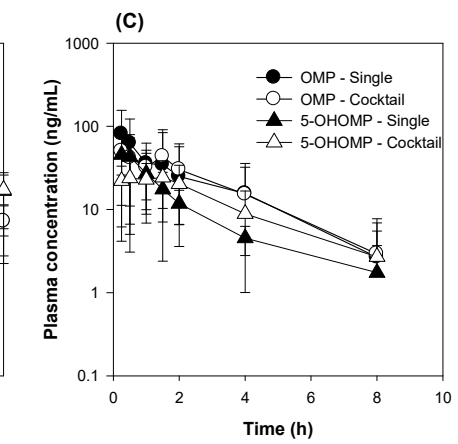

(E)
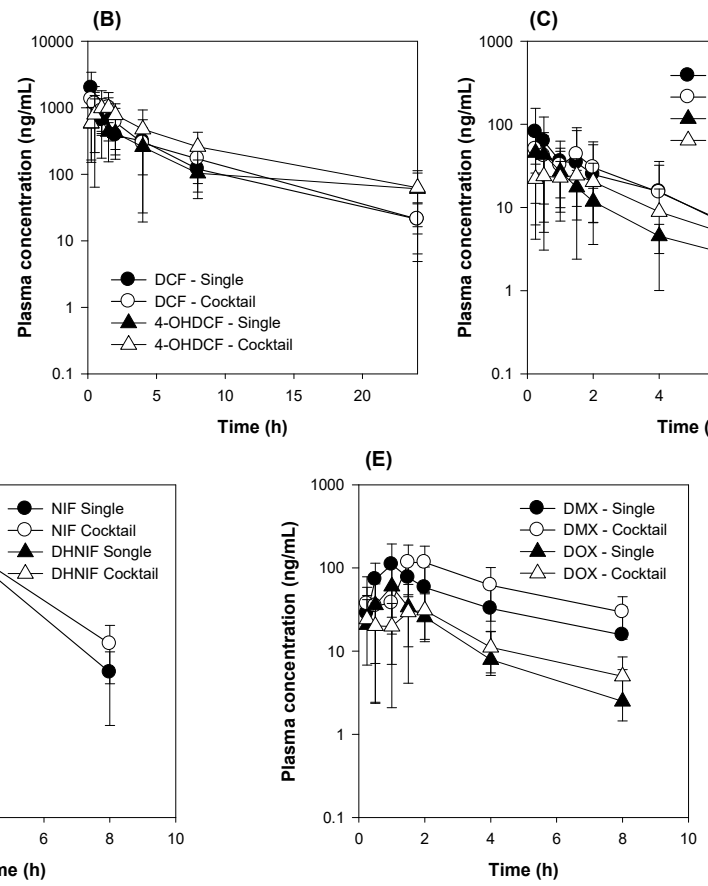

Figure 3. The plasma concentrations of (A) caffeine and paraxanthine (PXT), (B) diclofenac (DCF) and 4-hydroxy diclofenac (4-OHDCF), (C) omeprazole (OMP) and 5-hydroxyomeprazole (5-OHOMP), 
(D) nifedipine (NIF) and dehydronifedipine (DHNIF), and (E) dextromethorphan (DMX) and dextrorphan (DOX) following the single (closed symbol) and CYP cocktail (open symbol) oral administration of caffeine $(1 \mathrm{mg} / \mathrm{kg})$, diclofenac $(2 \mathrm{mg} / \mathrm{kg})$, omeprazole $(2 \mathrm{mg} / \mathrm{kg})$, nifedipine $(0.5 \mathrm{mg} / \mathrm{kg})$, and dextromethorphan $(10 \mathrm{mg} / \mathrm{kg})$ in rats. The data is expressed as the mean $\pm \operatorname{SD}(n=6)$.

Table 5. The comparison of pharmacokinetic parameters between single and CYP cocktail groups.

\begin{tabular}{|c|c|c|c|c|}
\hline Cyps & Probe Substrate & Parameters & Single $(n=6)$ & CYP Cocktail $(n=6)$ \\
\hline \multirow{6}{*}{ Cyp1a2 } & \multirow{3}{*}{ Caffeine } & $\mathrm{C}_{\max }(\mathrm{ng} / \mathrm{mL})$ & $866.9 \pm 310.8$ & $739.5 \pm 216.8$ \\
\hline & & $\mathrm{AUC}_{24 \mathrm{~h}}(\mathrm{ng} \cdot \mathrm{h} / \mathrm{mL})$ & $3042.8 \pm 1226.5$ & $3965.1 \pm 1632.2$ \\
\hline & & $\mathrm{AUC}_{\infty}(\mathrm{ng} \cdot \mathrm{h} / \mathrm{mL})$ & $3051.3 \pm 1231.0$ & $3991.4 \pm 1661.3$ \\
\hline & \multirow{3}{*}{ Paraxanthine } & $\mathrm{AUC}_{24 \mathrm{~h}}(\mathrm{ng} \cdot \mathrm{h} / \mathrm{mL})$ & $2878.6 \pm 931.3$ & $3593.8 \pm 1119.0$ \\
\hline & & $\mathrm{AUC}_{\infty}(\mathrm{ng} \cdot \mathrm{h} / \mathrm{mL})$ & $3135.4 \pm 985.6$ & $3152.4 \pm 471.4$ \\
\hline & & MR & $1.1 \pm 0.4$ & $1.0 \pm 0.4$ \\
\hline \multirow{6}{*}{ Cyp2c11 } & \multirow{3}{*}{ Diclofenac } & $\mathrm{C}_{\max }(\mathrm{ng} / \mathrm{mL})$ & $1863.9 \pm 1309.8$ & $1559.1 \pm 1036.2$ \\
\hline & & $\mathrm{AUC}_{24 \mathrm{~h}}(\mathrm{ng} \cdot \mathrm{h} / \mathrm{mL})$ & $4386.5 \pm 1264.3$ & $5505.2 \pm 1807.6$ \\
\hline & & $\mathrm{AUC}_{\infty}(\mathrm{ng} \cdot \mathrm{h} / \mathrm{mL})$ & $4552.2 \pm 1349.0$ & $5741.9 \pm 1836.8$ \\
\hline & \multirow{3}{*}{$\begin{array}{l}\text { 4-Hydroxy } \\
\text { diclofenac }\end{array}$} & $\mathrm{AUC}_{24 \mathrm{~h}}(\mathrm{ng} \cdot \mathrm{h} / \mathrm{mL})$ & $4080.0 \pm 2217.4$ & $6944.7 \pm 3761.8$ \\
\hline & & $\mathrm{AUC}_{\infty}(\mathrm{ng} \cdot \mathrm{h} / \mathrm{mL})$ & $5831.5 \pm 3529.0$ & $7844.7 \pm 4354.1$ \\
\hline & & MR & $1.0 \pm 0.6$ & $1.2 \pm 0.3$ \\
\hline \multirow{6}{*}{ Cyp2c11 } & \multirow{3}{*}{ Omeprazole } & $\mathrm{C}_{\max }(\mathrm{ng} / \mathrm{mL})$ & $182.3 \pm 158.5$ & $62.0 \pm 28.0$ \\
\hline & & $\mathrm{AUC}_{24 \mathrm{~h}}(\mathrm{ng} \cdot \mathrm{h} / \mathrm{mL})$ & $134.3 \pm 107.6$ & $145.1 \pm 87.0$ \\
\hline & & $\mathrm{AUC}_{\infty}(\mathrm{ng} \cdot \mathrm{h} / \mathrm{mL})$ & $134.5 \pm 107.8$ & $145.6 \pm 87.0$ \\
\hline & \multirow{3}{*}{$\begin{array}{l}\text { 5-Hydroxy } \\
\text { omeprazole }\end{array}$} & $\mathrm{AUC}_{24 \mathrm{~h}}(\mathrm{ng} \cdot \mathrm{h} / \mathrm{mL})$ & $116.6 \pm 69.7$ & $110.9 \pm 63.8$ \\
\hline & & $\mathrm{AUC}_{\infty}(\mathrm{ng} \cdot \mathrm{h} / \mathrm{mL})$ & $119.0 \pm 71.4$ & $112.0 \pm 64.6$ \\
\hline & & MR & $1.0 \pm 0.2$ & $0.8 \pm 0.1$ \\
\hline \multirow{6}{*}{ Сyp3a1 } & \multirow{3}{*}{ Nifedipine } & $\mathrm{C}_{\max }(\mathrm{ng} / \mathrm{mL})$ & $445.8 \pm 202.0$ & $420.1 \pm 224.5$ \\
\hline & & $\mathrm{AUC}_{24 \mathrm{~h}}(\mathrm{ng} \cdot \mathrm{h} / \mathrm{mL})$ & $1392.2 \pm 397.5$ & $1490.9 \pm 844.5$ \\
\hline & & $\mathrm{AUC}_{\infty}(\mathrm{ng} \cdot \mathrm{h} / \mathrm{mL})$ & $1393.8 \pm 397.0$ & $1499.6 \pm 841.9$ \\
\hline & \multirow{3}{*}{ Dehydronifedipine } & $\mathrm{AUC}_{24 \mathrm{~h}}(\mathrm{ng} \cdot \mathrm{h} / \mathrm{mL})$ & $22.3 \pm 5.8$ & $37.0 \pm 15.8$ \\
\hline & & $\mathrm{AUC}_{\infty}(\mathrm{ng} \cdot \mathrm{h} / \mathrm{mL})$ & $22.6 \pm 5.8$ & $37.4 \pm 15.9$ \\
\hline & & MR & $0.018 \pm 0.01$ & $0.028 \pm 0.01$ \\
\hline \multirow{6}{*}{ Cyp2d2 } & \multirow{3}{*}{ Dextromethorphan } & $\mathrm{C}_{\max }(\mathrm{ng} / \mathrm{mL})$ & $117.9 \pm 78.1$ & $180.0 \pm 136.2$ \\
\hline & & $\mathrm{AUC}_{24 \mathrm{~h}}(\mathrm{ng} \cdot \mathrm{h} / \mathrm{mL})$ & $457.5 \pm 373.3$ & $971.1 \pm 626.2$ \\
\hline & & $\mathrm{AUC}_{\infty}(\mathrm{ng} \cdot \mathrm{h} / \mathrm{mL})$ & $459.1 \pm 374.4$ & $1011.1 \pm 625.8$ \\
\hline & \multirow{3}{*}{ Dextrorphan } & $\mathrm{AUC}_{24 \mathrm{~h}}(\mathrm{ng} \cdot \mathrm{h} / \mathrm{mL})$ & $142.3 \pm 137.0$ & $196.9 \pm 214.6$ \\
\hline & & $\mathrm{AUC}_{\infty}(\mathrm{ng} \cdot \mathrm{h} / \mathrm{mL})$ & $208.6 \pm 145.3$ & $196.0 \pm 121.9$ \\
\hline & & MR & $0.30 \pm 0.16$ & $0.22 \pm 0.20$ \\
\hline
\end{tabular}

$\mathrm{C}_{\text {max }}$ : maximum concentration; $\mathrm{AUC}_{24 \mathrm{~h}}$ or $\mathrm{AUC}_{\infty}$ : area under the plasma concentration curve from zero to $24 \mathrm{~h}$ or infinite; MR: metabolic ratio calculated by dividing metabolite $\mathrm{AUC}_{24} \mathrm{~h}$ by parent $\mathrm{AUC}_{24} \mathrm{~h}$. The data expressed as the mean \pm standard deviation.

\subsection{The Comparison of Five Transporter Probe Substrates Administered Simultaneously versus Individually}

Next, we compared the plasma concentrations and pharmacokinetic parameters of the five probe substrates for transporters between the cocktail and single administration groups. Owing to the low bioavailability of probe substrates (less than 30\%), including digoxin, furosemide, metformin, valsartan, and methotrexate [13,22-26], probe substrates were intravenously administered and renal excretion was also measured since transporters, such as Octs, Oats, P-gp, and Mrp2 play significant roles in the renal excretion of their substrate drugs [11]. Figure 4A shows the plasma concentrations of metformin in the single and transporter cocktail groups, respectively. The plasma AUC values and 
renal excretion of metformin in the cocktail group were similar to the single administration group (Table 6), suggesting the remote interactions between cocktail and single administration in metformin pharmacokinetics. Similarly, the plasma concentrations of furosemide (Figure 4B), valsartan (Figure 4C), digoxin (Figure 4D), and methotrexate (Figure 4E) and their pharmacokinetic parameters (Table 6) were compared between the control and transporter cocktail groups. Table 4 reveals that the pharmacokinetic parameters of the probe substrates (i.e., furosemide, valsartan, digoxin, and methotrexate) in the transporter cocktail group were not statistically different in the single administration group: $p>0.05$ for all parameters according to the Mann-Whitney $U$ test. Collectively, these results suggest that the five transporter cocktail substrates could be concomitantly administered to rats for the evaluation of the transporter activity and substrate drug pharmacokinetics. The cocktail that included metformin $(0.5 \mathrm{mg} / \mathrm{kg})$, furosemide $(0.1 \mathrm{mg} / \mathrm{kg})$, valsartan $(0.2 \mathrm{mg} / \mathrm{kg})$, digoxin $(2 \mathrm{mg} / \mathrm{kg})$, and methotrexate $(0.5 \mathrm{mg} / \mathrm{kg})$ was intravenously administered.
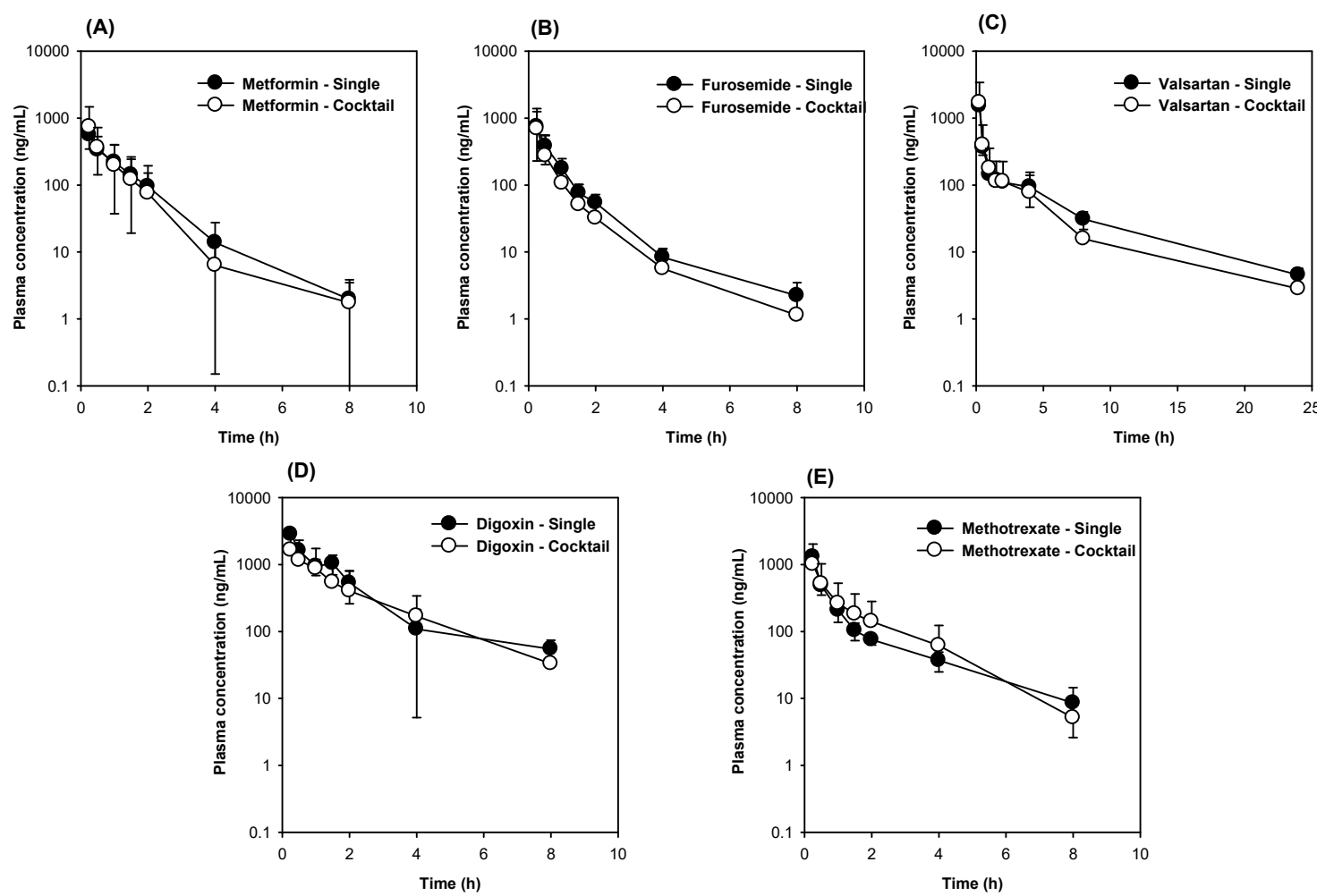

Figure 4. The plasma concentrations of (A) metformin, (B) furosemide, (C) valsartan, (D) digoxin, and (E) methotrexate following the single (closed symbol) and transporter cocktail (open symbol) administration of metformin $(0.5 \mathrm{mg} / \mathrm{kg})$, furosemide $(0.1 \mathrm{mg} / \mathrm{kg})$, valsartan $(0.2 \mathrm{mg} / \mathrm{kg})$, digoxin $(2 \mathrm{mg} / \mathrm{kg})$, and methotrexate $(0.5 \mathrm{mg} / \mathrm{kg})$ intravenous injection in rats. The data are presented as the means $\pm \mathrm{SD}$ $(n=6)$.

\subsection{The Comparison of Pharmacokinetics Parameters Following Administration of Dual Cocktail vs. Five} Transporter or Five CYP Cocktail

Next, we compared the pharmacokinetic parameters of the 10 probe substrates following the concomitant administration of an oral dose of five CYP cocktail and intravenous injection of five transporter cocktail in rats that received either the five CYP cocktail set or five transporter cocktail set, which were shown to be free of any interactions between individual probes (Figures 3 and 4).

No significant difference was observed in the major pharmacokinetic parameters of 10 probe substrates administered as a separate five-CYP cocktail or five-transporter cocktail and dual cocktail (Figure 5 and Table 7). The results suggested that the administration of 10 probe substrates as dual cocktail could be used to phenotype the in vivo CYP or transporter activity or to evaluate potential DDIs. 
Table 6. The comparison of pharmacokinetic parameters between single and transporter cocktail groups.

\begin{tabular}{|c|c|c|c|c|}
\hline Transporters & Probe Substrate & Parameters & Single $(n=6)$ & $\begin{array}{c}\text { Transporter } \\
\text { Cocktail }(n=6)\end{array}$ \\
\hline Oct1/2 & Metformin & $\begin{array}{c}\mathrm{AUC}_{24 \mathrm{~h}}(\mathrm{ng} \cdot \mathrm{h} / \mathrm{mL}) \\
\mathrm{AUC}_{\infty}(\mathrm{ng} \cdot \mathrm{h} / \mathrm{mL}) \\
\mathrm{Xu}(\mathrm{ng})\end{array}$ & $\begin{array}{c}733 \pm 402 \\
750 \pm 415 \\
47,806 \pm 29,071\end{array}$ & $\begin{aligned} 767 & \pm 245 \\
777 & \pm 247 \\
52,474 & \pm 19,441\end{aligned}$ \\
\hline Oat1/3 & Furosemide & $\begin{array}{c}\mathrm{AUC}_{24 \mathrm{~h}}(\mathrm{ng} \cdot \mathrm{h} / \mathrm{mL}) \\
\mathrm{AUC}_{\infty}(\mathrm{ng} \cdot \mathrm{h} / \mathrm{mL}) \\
\text { Xu }(\mathrm{ng})\end{array}$ & $\begin{array}{c}766 \pm 370 \\
773 \pm 366 \\
2330 \pm 1285\end{array}$ & $\begin{array}{c}652 \pm 130 \\
654 \pm 130 \\
6969 \pm 3330\end{array}$ \\
\hline Oatp2 & Valsartan & $\begin{array}{c}\mathrm{AUC}_{24 \mathrm{~h}}(\mathrm{ng} \cdot \mathrm{h} / \mathrm{mL}) \\
\mathrm{AUC}_{\infty}(\mathrm{ng} \cdot \mathrm{h} / \mathrm{mL}) \\
\text { Xu (ng) }\end{array}$ & $\begin{array}{c}2252 \pm 247 \\
2285 \pm 245 \\
920 \pm 504\end{array}$ & $\begin{array}{c}2198 \pm 518 \\
2225 \pm 519 \\
627 \pm 441\end{array}$ \\
\hline P-gp & Digoxin & $\begin{array}{c}\mathrm{AUC}_{24 \mathrm{~h}}(\mathrm{ng} \cdot \mathrm{h} / \mathrm{mL}) \\
\mathrm{AUC}_{\infty}(\mathrm{ng} \cdot \mathrm{h} / \mathrm{mL}) \\
\mathrm{Xu}(\mathrm{ng})\end{array}$ & $\begin{array}{c}4079 \pm 716 \\
4232 \pm 828 \\
29,954 \pm 11,498\end{array}$ & $\begin{array}{c}3151 \pm 1009 \\
3193 \pm 1011 \\
23,444 \pm 10,659\end{array}$ \\
\hline Mrp2 & Methotrexate & $\begin{array}{c}\mathrm{AUC}_{24} \mathrm{~h}(\mathrm{ng} \cdot \mathrm{h} / \mathrm{mL}) \\
\mathrm{AUC}_{\infty}(\mathrm{ng} \cdot \mathrm{h} / \mathrm{mL}) \\
\mathrm{Xu}(\mathrm{ng})\end{array}$ & $\begin{array}{c}1339 \pm 242 \\
1362 \pm 264 \\
2604 \pm 1924\end{array}$ & $\begin{array}{c}1319 \pm 252 \\
1329 \pm 260 \\
4621 \pm 1431\end{array}$ \\
\hline
\end{tabular}

$\mathrm{AUC}_{24 \mathrm{~h}}$ or $\mathrm{AUC}_{\infty}$ : area under the plasma concentration curve from zero to $24 \mathrm{~h}$ or infinite; $\mathrm{Xu}$ : excreted amount of substrate into urine for $24 \mathrm{~h}$. The data expressed as the mean \pm standard deviation.
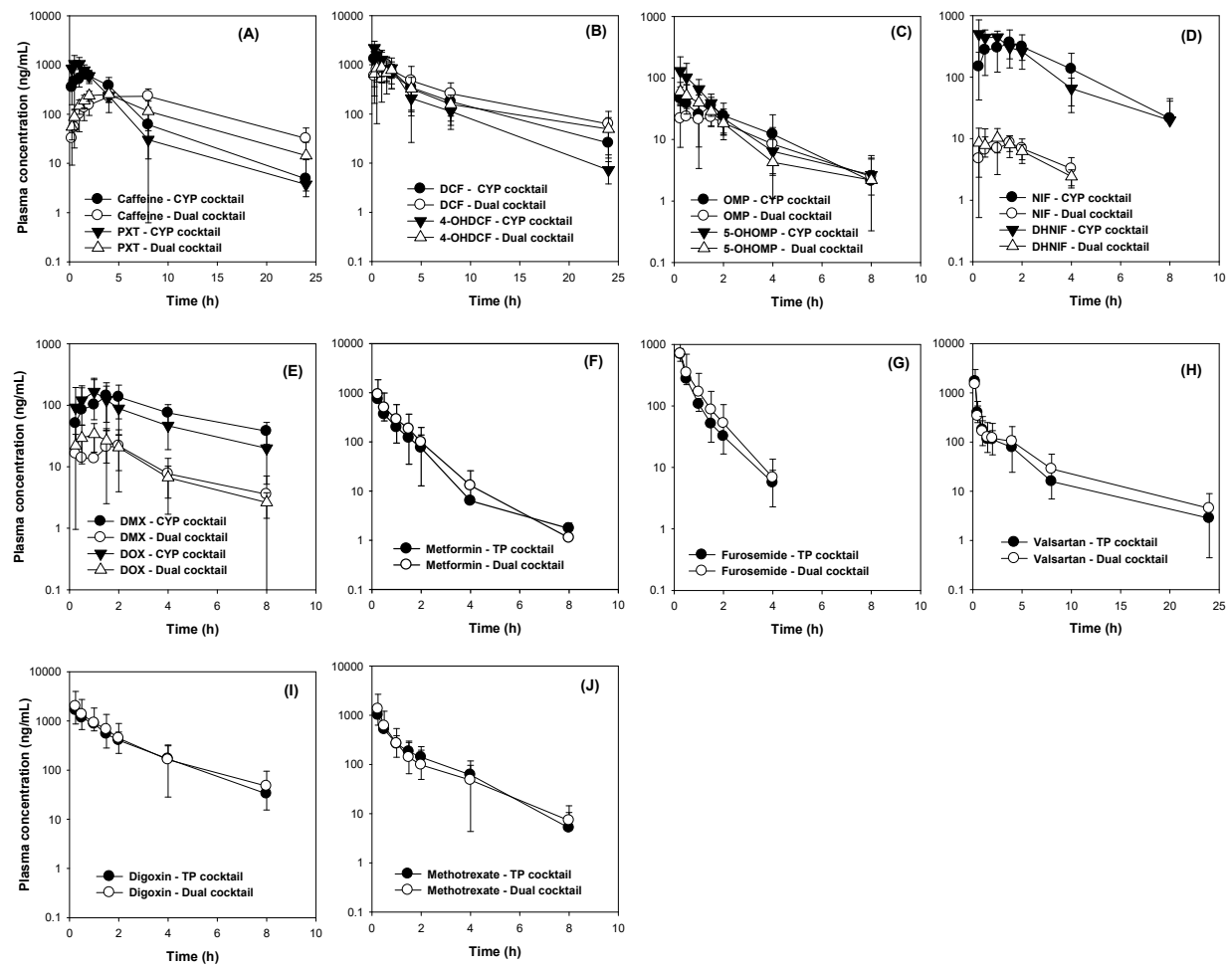

Figure 5. The plasma concentrations of (A) caffeine and paraxanthine (PXT), (B) diclofenac (DCF) and 4-hydroxy diclofenac (4-OHDCF), (C) omeprazole (OMP) and 5-hydroxyomeprazole (5-OHOMP), (D) nifedipine (NIF) and dehydronifedipine (DHNIF), and (E) dextromethorphan (DMX) and dextrorphan (DOX) following the administration of five CYP cocktail (closed symbol) and dual cocktail (open symbol) in rats $(n=6)$. The plasma concentrations of $(\mathbf{F})$ metformin, $(\mathbf{G})$ furosemide, $(\mathbf{H})$ valsartan, (I) digoxin, and (J) methotrexate following the administration of transporter (TP) cocktail (closed symbol) and dual cocktail (open symbol) in rats $(n=6)$. The CYP cocktail consists of caffeine $(1 \mathrm{mg} / \mathrm{kg})$, diclofenac $(2 \mathrm{mg} / \mathrm{kg})$, omeprazole $(2 \mathrm{mg} / \mathrm{kg})$, nifedipine $(0.5 \mathrm{mg} / \mathrm{kg})$, and dextromethorphan $(10 \mathrm{mg} / \mathrm{kg})$ and orally administered. The TP cocktail consists of metformin $(0.5 \mathrm{mg} / \mathrm{kg})$, furosemide $(0.1 \mathrm{mg} / \mathrm{kg})$, valsartan $(0.2 \mathrm{mg} / \mathrm{kg})$, digoxin $(2 \mathrm{mg} / \mathrm{kg})$, and methotrexate $(0.5 \mathrm{mg} / \mathrm{kg})$ and administered via intravenous injection. Dual cocktail is a mixture of CYP and TP cocktails. The data are presented as the means \pm SD ( $n=6$ for CYP cocktail or transporter cocktail, $n=7$ for dual cocktail). 
Table 7. The comparison of pharmacokinetic parameters between the dual cocktail and CYP or transporter cocktail groups.

\begin{tabular}{|c|c|c|c|c|}
\hline Cyps/Transporters & Probe Substrate & Parameters & $\begin{array}{l}\text { CYP or Transporter } \\
\text { Cocktail }(n=6)\end{array}$ & Dual Cocktail $(n=7)$ \\
\hline \multirow{5}{*}{ Cyp1a2 } & \multirow{2}{*}{ Caffeine } & $\mathrm{AUC}_{24 \mathrm{~h}}(\mathrm{ng} \cdot \mathrm{h} / \mathrm{mL})$ & $3965 \pm 1632$ & $3533 \pm 1788$ \\
\hline & & $\mathrm{AUC}_{\infty}(\mathrm{ng} \cdot \mathrm{h} / \mathrm{mL})$ & $3991 \pm 1661$ & $3580 \pm 1889$ \\
\hline & \multirow{3}{*}{ Paraxanthine } & $\mathrm{AUC}_{24 \mathrm{~h}}(\mathrm{ng} \cdot \mathrm{h} / \mathrm{mL})$ & $3593.83 \pm 1119.05$ & $2416.56 \pm 1073.93$ \\
\hline & & $\mathrm{AUC}_{\infty}(\mathrm{ng} \cdot \mathrm{h} / \mathrm{mL})$ & $3152.35 \pm 471.42$ & $2573.44 \pm 1125.72$ \\
\hline & & MR & $1.01 \pm 0.37$ & $0.77 \pm 0.17$ \\
\hline \multirow{5}{*}{ Сур2c11 } & \multirow{2}{*}{ Diclofenac } & $\mathrm{AUC}_{24 \mathrm{~h}}(\mathrm{ng} \cdot \mathrm{h} / \mathrm{mL})$ & $5505 \pm 1808$ & $5073 \pm 1186$ \\
\hline & & $\mathrm{AUC}_{\infty}(\mathrm{ng} \cdot \mathrm{h} / \mathrm{mL})$ & $5742 \pm 1837$ & $5116 \pm 1200$ \\
\hline & \multirow{3}{*}{ 4-Hydroxy diclofenac } & $\mathrm{AUC}_{24 \mathrm{~h}}(\mathrm{ng} \cdot \mathrm{h} / \mathrm{mL})$ & $6944.71 \pm 3761.81$ & $5184.02 \pm 1609.43$ \\
\hline & & $\mathrm{AUC}_{\infty}(\mathrm{ng} \cdot \mathrm{h} / \mathrm{mL})$ & $7844.69 \pm 4354.05$ & $5707.53 \pm 2017.07$ \\
\hline & & MR & $1.20 \pm 0.34$ & $1.02 \pm 0.13$ \\
\hline \multirow{5}{*}{ Сур2c11 } & \multirow{2}{*}{ Omeprazole } & $\mathrm{AUC}_{24 \mathrm{~h}}(\mathrm{ng} \cdot \mathrm{h} / \mathrm{mL})$ & $145 \pm 87$ & $191 \pm 53$ \\
\hline & & $\mathrm{AUC}_{\infty}(\mathrm{ng} \cdot \mathrm{h} / \mathrm{mL})$ & $146 \pm 87$ & $194 \pm 52$ \\
\hline & \multirow{3}{*}{ 5-Hydroxyomeprazole } & $\mathrm{AUC}_{24 \mathrm{~h}}(\mathrm{ng} \cdot \mathrm{h} / \mathrm{mL})$ & $110.93 \pm 63.79$ & $147.55 \pm 68.57$ \\
\hline & & $\mathrm{AUC}_{\infty}(\mathrm{ng} \cdot \mathrm{h} / \mathrm{mL})$ & $111.99 \pm 64.62$ & $150.42 \pm 67.29$ \\
\hline & & MR & $0.76 \pm 0.08$ & $0.74 \pm 0.14$ \\
\hline \multirow{5}{*}{ Сур3а4 } & \multirow{2}{*}{ Nifedipine } & $\mathrm{AUC}_{24 \mathrm{~h}}(\mathrm{ng} \cdot \mathrm{h} / \mathrm{mL})$ & $1491 \pm 844$ & $1320 \pm 206$ \\
\hline & & $\mathrm{AUC}_{\infty}(\mathrm{ng} \cdot \mathrm{h} / \mathrm{mL})$ & $1500 \pm 842$ & $1343 \pm 204$ \\
\hline & \multirow{3}{*}{ Dehydronifedipine } & $\mathrm{AUC}_{24 \mathrm{~h}}(\mathrm{ng} \cdot \mathrm{h} / \mathrm{mL})$ & $37.03 \pm 15.84$ & $35.02 \pm 10.94$ \\
\hline & & $\mathrm{AUC}_{\infty}(\mathrm{ng} \cdot \mathrm{h} / \mathrm{mL})$ & $37.37 \pm 15.90$ & $36.99 \pm 10.69$ \\
\hline & & MR & $0.028 \pm 0.012$ & $0.025 \pm 0.008$ \\
\hline \multirow{5}{*}{ Сyp2d2 } & \multirow{2}{*}{ Dextromethorphan } & $\mathrm{AUC}_{24 \mathrm{~h}}(\mathrm{ng} \cdot \mathrm{h} / \mathrm{mL})$ & $971 \pm 626$ & $654 \pm 406$ \\
\hline & & $\mathrm{AUC}_{\infty}(\mathrm{ng} \cdot \mathrm{h} / \mathrm{mL})$ & $1011 \pm 626$ & $674 \pm 387$ \\
\hline & \multirow{3}{*}{ Dextrorphan } & $\mathrm{AUC}_{24 \mathrm{~h}}(\mathrm{ng} \cdot \mathrm{h} / \mathrm{mL})$ & $196.90 \pm 214.62$ & $155.63 \pm 69.83$ \\
\hline & & $\mathrm{AUC}_{\infty}(\mathrm{ng} \cdot \mathrm{h} / \mathrm{mL})$ & $196.02 \pm 121.90$ & $213.90 \pm 74.20$ \\
\hline & & MR & $0.20 \pm 0.22$ & $0.26 \pm 0.08$ \\
\hline \multirow{3}{*}{ Oct1/2 } & \multirow{3}{*}{ Metformin } & $\mathrm{AUC}_{24 \mathrm{~h}}(\mathrm{ng} \cdot \mathrm{h} / \mathrm{mL})$ & $767 \pm 245$ & $1027 \pm 349$ \\
\hline & & $\mathrm{AUC}_{\infty}(\mathrm{ng} \cdot \mathrm{h} / \mathrm{mL})$ & $777 \pm 247$ & $1032 \pm 348$ \\
\hline & & $\mathrm{Xu}$ (ng) & $52,474 \pm 19,441$ & $48,885 \pm 17,369$ \\
\hline \multirow{3}{*}{ Oat1/3 } & \multirow{3}{*}{ Furosemide } & $\mathrm{AUC}_{24 \mathrm{~h}}(\mathrm{ng} \cdot \mathrm{h} / \mathrm{mL})$ & $652 \pm 130$ & $701 \pm 175$ \\
\hline & & $\mathrm{AUC}_{\infty}(\mathrm{ng} \cdot \mathrm{h} / \mathrm{mL})$ & $654 \pm 130$ & $704 \pm 174$ \\
\hline & & Xu (ng) & $6969 \pm 3330$ & $4950 \pm 2211$ \\
\hline \multirow{3}{*}{ Oatp2 } & \multirow{3}{*}{ Valsartan } & $\mathrm{AUC}_{24 \mathrm{~h}}(\mathrm{ng} \cdot \mathrm{h} / \mathrm{mL})$ & $2198 \pm 518$ & $2258 \pm 674$ \\
\hline & & $\mathrm{AUC}_{\infty}(\mathrm{ng} \cdot \mathrm{h} / \mathrm{mL})$ & $2225 \pm 519$ & $2289 \pm 667$ \\
\hline & & Xu (ng) & $627 \pm 441$ & $516 \pm 338$ \\
\hline \multirow{3}{*}{ P-gp } & \multirow{3}{*}{ Digoxin } & $\mathrm{AUC}_{24 \mathrm{~h}}(\mathrm{ng} \cdot \mathrm{h} / \mathrm{mL})$ & $3151 \pm 1009$ & $3302 \pm 717$ \\
\hline & & $\mathrm{AUC}_{\infty}(\mathrm{ng} \cdot \mathrm{h} / \mathrm{mL})$ & $3193 \pm 1011$ & $3430 \pm 727$ \\
\hline & & $\mathrm{Xu}$ (ng) & $23,444 \pm 10,659$ & $18,542 \pm 7040$ \\
\hline \multirow{3}{*}{ Mrp2 } & \multirow{3}{*}{ Methotrexate } & $\mathrm{AUC}_{24 \mathrm{~h}}(\mathrm{ng} \cdot \mathrm{h} / \mathrm{mL})$ & $1319 \pm 252$ & $1432 \pm 414$ \\
\hline & & $\mathrm{AUC}_{\infty}(\mathrm{ng} \cdot \mathrm{h} / \mathrm{mL})$ & $1329 \pm 260$ & $1451 \pm 421$ \\
\hline & & Xu (ng) & $4621 \pm 1431$ & $5680 \pm 3451$ \\
\hline
\end{tabular}

$\mathrm{AUC}_{24 \mathrm{~h}}$ or $\mathrm{AUC}_{\infty}$ : area under the plasma concentration curve from zero to $24 \mathrm{~h}$ or infinite; MR: metabolic ratio calculated by dividing metabolite $\mathrm{AUC}_{24} \mathrm{~h}$ by parent $\mathrm{AUC}_{24} \mathrm{~h} ; \mathrm{Xu}$ : excreted amount of substrate into urine for $24 \mathrm{~h}$. The data expressed as the mean \pm standard deviation.

\subsection{Dual Cocktail Validation by Evaluating the Effects of Known CYP or Transporter Modulators on the} Pharmacokinetic Parameters of Probe Substrates

To validate the dual cocktail set, a single oral dose of ketoconazole (10 $\mathrm{mg} / \mathrm{kg})$ as a representative Cyp3a inhibitor [51], a single oral dose of rifampin $(20 \mathrm{mg} / \mathrm{kg})$ as a representative Oatp inhibitor [21], 
and multiple oral doses of rifampin ( $20 \mathrm{mg} / \mathrm{kg} / \mathrm{dy}$ for five days) as a representative Cyp3a and P-gp inducer [52,53] were administered before the dual cocktail substrates. Rifampin and ketoconazole has been widely used to investigate the pharmacokinetic DDIs in human as well as in animals [54,55].

As shown in Table 8, a single oral dose of ketoconazole significantly increased the plasma concentrations of nifedipine and omeprazole and consequently decreased the MR of nifedipine and omeprazole. Other substrates were not significantly changed by ketoconazole co-administration. The single oral administration of rifampin selectively inhibited the valsartan disposition and, therefore, increased the plasma exposure of valsartan by 3.5-fold, although the AUC values of other probe substrates and MR and AUC values of metabolites in the dual cocktail were not changed by the single rifampin treatment (Table 8), suggesting the selectivity of Oatp inhibition by single rifampin treatment and the feasibility of the dual cocktail in assessing pharmacokinetic DDIs. Repeated rifampin administration, which is known for inducing Сур3a and P-gp [52,53], increased the disposition of nifedipine and digoxin, which consequently decreased the AUC values of nifedipine and digoxin (Table 8). The AUC values of other substrate drugs were not altered by the repeated rifampin administration.

In summary, the dual cocktail of 10 probe substrates for five CYPs and five transporters was successfully developed without any significant pharmacokinetic DDIs among the probe substrates. This dual cocktail was validated using a representative Сyp3a inhibitor ketoconazole, an Oatp inhibitor rifampin (single administration) [21], and a repeated rifampin administration, which acts as a Cyp3a and P-gp inducer $[52,53]$.

However, the AUC values of dehydronifedipine were not significantly changed by the repeated rifampin treatment, and the AUC values of the metabolites and MR of nifedipine might not indicate Cyp3a activity modulation. In omeprazole and dextromethorphan cases, the MR of these substrates could be compromised owing to multiple enzyme involvement in their metabolism [56,57]. Moreover, since the major elimination route of valsartan and methotrexate is biliary excretion [20,58,59], Xu could not represent the transport activity of Octs, Oatps, P-gp, and Mrp2. Therefore, the phenotypic index for the assessment of potential pharmacokinetic DDIs could be suggested as the changes in the AUC values of probe substrates (Table 9).

\subsection{The Use of the Dual Cocktail to Assess the Pharmacokinetic Herb-Drug Interaction of RGE Treatment}

To further validate the utility of the dual cocktail, the rats were pretreated with RGE, and the dual cocktail was administered to assess the pharmacokinetic herb-drug interactions between probe substrates and RGE following the oral administration of RGE ( $1.4 \mathrm{~g} / \mathrm{kg} /$ day) for seven days.

The plasma concentrations of probe substrates are shown in Figure 6. The plasma concentration profiles of caffeine, dichlofenac, omeprazole, dextromethorphan, valsartan, and digoxin in the RGE group were similar to those in the control group. However, the plasma concentrations of metformin, furosemide, and methotrexate in the RGE group were higher than those in the control group (Figure 6).

The comparison of AUC values of the probe substrates between the control and RGE groups showed no significant pharmacokinetic herb-drug interaction involving caffeine, dichlofenac, omeprazole, dextromethorphan, valsartan, and digoxin after the repeated RGE treatment. However, the AUC values of nifedipine, metformin, furosemide, and methotrexate following the RGE treatment were significantly greater than the control group (Figure 7). The results suggested that the repeated RGE administration decreased the activity of Сyp3a, Oct, Oat, and Mrp2, and subsequently decreased the disposition of their specific probe substrate drugs, including nifedipine, metformin, furosemide, and methotrexate, respectively. The observed pharmacokinetic interactions between RGE and metformin and methotrexate are consistent with previous results [12,21]. The lack of pharmacokinetic interaction between valsartan and RGE is also consistent with previous reports [21]. 
Table 8. The effect of ketoconazole and rifampin on the pharmacokinetic parameters of probe substrates of the dual cocktail and metabolites.

\begin{tabular}{|c|c|c|c|c|c|c|c|}
\hline \multirow[b]{2}{*}{ Cyps/Transporters } & \multirow[b]{2}{*}{ Probe Substrate } & \multirow[b]{2}{*}{ Parameters } & \multicolumn{3}{|c|}{ Inhibition } & \multicolumn{2}{|c|}{ Induction } \\
\hline & & & $\begin{array}{c}\text { Control } \\
(n=6)\end{array}$ & $\begin{array}{c}\text { Ketoconazole } \\
(n=4)\end{array}$ & $\begin{array}{c}\text { Rifampin } \\
(n=4)\end{array}$ & $\begin{array}{l}\text { Control } \\
(n=4)\end{array}$ & $\begin{array}{c}\text { Rifampin } \\
(n=4)\end{array}$ \\
\hline \multirow{3}{*}{ Cyp1a2 } & Caffeine & $\mathrm{AUC}_{24 \mathrm{~h}}(\mathrm{ng} \cdot \mathrm{h} / \mathrm{mL})$ & $3104.8 \pm 963.8$ & $3579.8 \pm 891.5$ & $4131.9 \pm 2129.2$ & $3959.0 \pm 1824.8$ & $3687.9 \pm 540.0$ \\
\hline & \multirow{2}{*}{ Paraxanthine } & $\mathrm{AUC}_{24 \mathrm{~h}}(\mathrm{ng} \cdot \mathrm{h} / \mathrm{mL})$ & $2416.6 \pm 1073.9$ & $3138.1 \pm 1042.8$ & $3555.2 \pm 1740.2$ & $3248.6 \pm 819.6$ & $3107.9 \pm 351.6$ \\
\hline & & MR & $0.8 \pm 0.2$ & $0.9 \pm 0.1$ & $0.9 \pm 0.0$ & $0.9 \pm 0.4$ & $0.9 \pm 0.1$ \\
\hline \multirow{3}{*}{ Сур2c11 } & Diclofenac & $\mathrm{AUC}_{24 \mathrm{~h}}(\mathrm{ng} \cdot \mathrm{h} / \mathrm{mL})$ & $3551.7 \pm 887.7$ & $3863.3 \pm 1109.8$ & $3681.9 \pm 724.2$ & $4980.0 \pm 1419.7$ & $5744.9 \pm 1647.1$ \\
\hline & \multirow{2}{*}{ 4-Hydroxy diclofenac } & $\mathrm{AUC}_{24 \mathrm{~h}}(\mathrm{ng} \cdot \mathrm{h} / \mathrm{mL})$ & $3480.8 \pm 1062.3$ & $3584.7 \pm 1222.2$ & $3024.5 \pm 459.7$ & $5848.2 \pm 2944.7$ & $3986.8 \pm 5627.2$ \\
\hline & & MR & $1.0 \pm 0.2$ & $0.9 \pm 0.2$ & $0.8 \pm 0.2$ & $1.1 \pm 0.3$ & $0.6 \pm 0.6$ \\
\hline \multirow{3}{*}{ Сур2c11 } & Omeprazole & $\mathrm{AUC}_{24 \mathrm{~h}}(\mathrm{ng} \cdot \mathrm{h} / \mathrm{mL})$ & $191.3 \pm 53.3$ & $403.5 \pm 116.0 *$ & $217.4 \pm 18.3$ & $207.9 \pm 43.1$ & $245.2 \pm 31.2$ \\
\hline & \multirow{2}{*}{ 5-Hydroxyomeprazole } & $\mathrm{AUC}_{24 \mathrm{~h}}(\mathrm{ng} \cdot \mathrm{h} / \mathrm{mL})$ & $119.0 \pm 27.5$ & $121.4 \pm 37.1$ & $105.0 \pm 24.6$ & $129.0 \pm 17.8$ & $133.4 \pm 26.9$ \\
\hline & & MR & $0.6 \pm 0.1$ & $0.3 \pm 0.0 *$ & $0.5 \pm 0.1$ & $0.6 \pm 0.1$ & $0.6 \pm 0.1$ \\
\hline \multirow{3}{*}{ Сур3a1 } & Nifedipine & $\mathrm{AUC}_{24 \mathrm{~h}}(\mathrm{ng} \cdot \mathrm{h} / \mathrm{mL})$ & $1367.3 \pm 201.1$ & $5021.9 \pm 3577.3^{*}$ & $1597.3 \pm 187.6$ & $1595.2 \pm 648.7$ & $859.0 \pm 268.2 *$ \\
\hline & \multirow{2}{*}{ Dehydronifedipine } & $\mathrm{AUC}_{24 \mathrm{~h}}(\mathrm{ng} \cdot \mathrm{h} / \mathrm{mL})$ & $60.2 \pm 16.4$ & $39.2 \pm 11.1^{*}$ & $67.5 \pm 4.6$ & $40.6 \pm 14.7$ & $56.0 \pm 13.1$ \\
\hline & & MR & $0.05 \pm 0.02$ & $0.01 \pm 0.00$ * & $0.04 \pm 0.00$ & $0.0 \pm 0.0$ & $0.1 \pm 0.0 *$ \\
\hline \multirow{3}{*}{ Cyp2d2 } & Dextromethorphan & $\mathrm{AUC}_{24 \mathrm{~h}}(\mathrm{ng} \cdot \mathrm{h} / \mathrm{mL})$ & $1002.4 \pm 206.0$ & $1170.3 \pm 228.8$ & $1029.7 \pm 455.1$ & $1117.4 \pm 574.1$ & $970.1 \pm 188.4$ \\
\hline & \multirow{2}{*}{ Dextrorphan } & $\mathrm{AUC}_{24 \mathrm{~h}}(\mathrm{ng} \cdot \mathrm{h} / \mathrm{mL})$ & $234.5 \pm 167.5$ & $142.5 \pm 27.0$ & $230.6 \pm 43.1$ & $229.2 \pm 223.0$ & $361.9 \pm 205.8$ \\
\hline & & MR & $0.2 \pm 0.2$ & $0.1 \pm 0.0$ & $0.2 \pm 0.1$ & $0.2 \pm 0.2$ & $0.4 \pm 0.2$ \\
\hline Oct1/2 & Metformin & $\mathrm{AUC}_{24 \mathrm{~h}}(\mathrm{ng} \cdot \mathrm{h} / \mathrm{mL})$ & $908.0 \pm 159.7$ & $980.2 \pm 90.3$ & $788.1 \pm 127.4$ & $976.3 \pm 406.4$ & $1294.9 \pm 468.0$ \\
\hline Oat1/3 & Furosemide & $\mathrm{AUC}_{24 \mathrm{~h}}(\mathrm{ng} \cdot \mathrm{h} / \mathrm{mL})$ & $700.9 \pm 175.2$ & $731.5 \pm 123.6$ & $682.6 \pm 76.2$ & $614.5 \pm 83.9$ & $524.2 \pm 28.2$ \\
\hline Oatp2 & Valsartan & $\mathrm{AUC}_{24 \mathrm{~h}}(\mathrm{ng} \cdot \mathrm{h} / \mathrm{mL})$ & $2488.5 \pm 315.6$ & $2227.7 \pm 1007.3$ & $8693.9 \pm 936.1^{*}$ & $2258.4 \pm 673.5$ & $2333.4 \pm 137.5$ \\
\hline P-gp & Digoxin & $\mathrm{AUC}_{24 \mathrm{~h}}(\mathrm{ng} \cdot \mathrm{h} / \mathrm{mL})$ & $3302.0 \pm 717.5$ & $3677.2 \pm 745.8$ & $3732.4 \pm 220.8$ & $3302.0 \pm 717.5$ & $1349.5 \pm 738.9$ * \\
\hline Mrp2 & Methotrexate & $\mathrm{AUC}_{24 \mathrm{~h}}(\mathrm{ng} \cdot \mathrm{h} / \mathrm{mL})$ & $1432.1 \pm 413.8$ & $1229.0 \pm 498.4$ & $1332.9 \pm 463.9$ & $1432.1 \pm 413.8$ & $1294.0 \pm 484.0$ \\
\hline
\end{tabular}

$\mathrm{AUC}_{24 \mathrm{~h}}$ : area under the plasma concentration curve from zero to $24 \mathrm{~h}$; MR: metabolic ratio calculated by dividing metabolite $\mathrm{AUC}_{24} \mathrm{~h}$ by parent $\mathrm{AUC} \mathrm{C}_{24} \mathrm{~h} * p<0.05$ statistically significant compared with control group by Mann-Whitney $U$ test. The data expressed as the mean \pm standard deviation. 
Table 9. The composition and phenotypic index of dual cocktail.

\begin{tabular}{ccccc}
\hline Category & $\begin{array}{c}\text { Isoforms in Rat } \\
\text { (Human) }\end{array}$ & $\begin{array}{c}\text { Probe Substrates } \\
\text { (Dose, } \mathbf{m g} / \mathbf{k g})\end{array}$ & $\begin{array}{c}\text { Route of } \\
\text { Administration }\end{array}$ & Phenotypic Index \\
\hline \multirow{5}{*}{ CYPs } & Cyp1a2 (CYP1A2) & Caffeine $(1 \mathrm{mg} / \mathrm{kg})$ & & AUC \\
& Cyp2c11 (CYP2C9) & Diclofenac $(2 \mathrm{mg} / \mathrm{kg})$ & AUC \\
& Cyp2c11 (CYP2C19) & Omeprazole $(2 \mathrm{mg} / \mathrm{kg})$ & PO & AUC \\
& Cyp2d2 (CYP2D6) & $\begin{array}{c}\text { Dextromethorphan }(10 \mathrm{mg} / \mathrm{kg}) \\
\text { Nifedipine }(0.5 \mathrm{mg} / \mathrm{kg})\end{array}$ & AUC \\
& Cyp3a1 (CYP3A4) & AUC \\
\hline \multirow{5}{*}{ Transporters } & Metformin $(0.5 \mathrm{mg} / \mathrm{kg})$ & & AUC \\
& Oct1/2 (OCT1/2) & Fut1/3 (OAT1/3) & Valsemide $(0.1 \mathrm{mg} / \mathrm{kg})$ & AUC \\
& Oatp2 (OATP1B1/1B3) & Dalsan $(0.2 \mathrm{mg} / \mathrm{kg})$ & AV \\
& P-gp (P-gp) & Digoxin $(2 \mathrm{mg} / \mathrm{kg})$ & AUC \\
\hline
\end{tabular}

(A) Caffeine

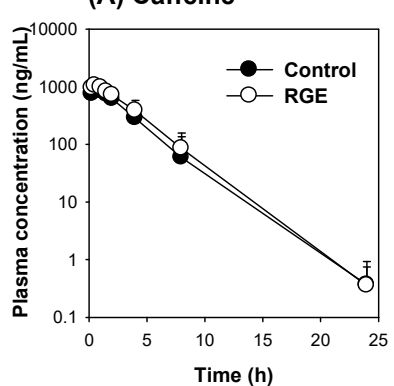

(E) DMX

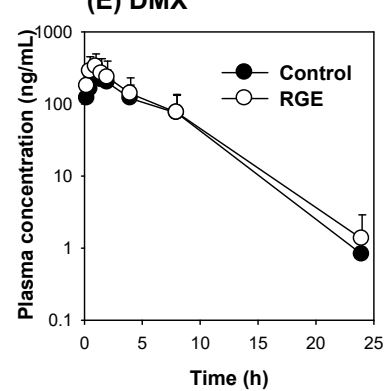

(I) Digoxin

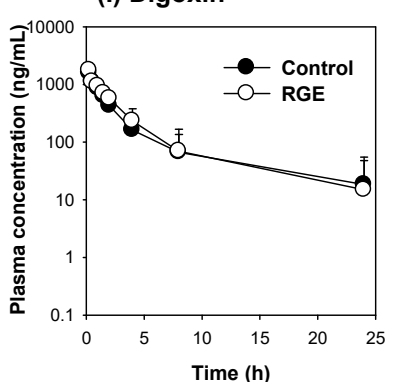

(B) DCF

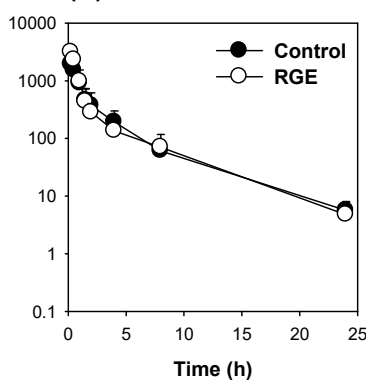

(F) Metformin

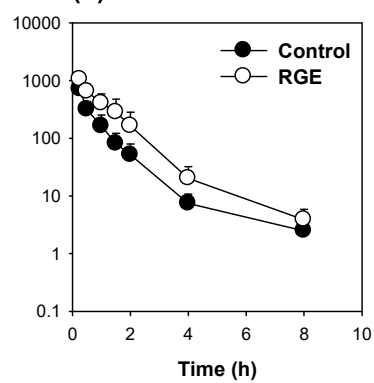

(J) Methtrexate

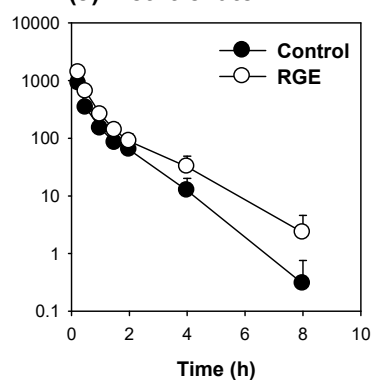

(C) OMP

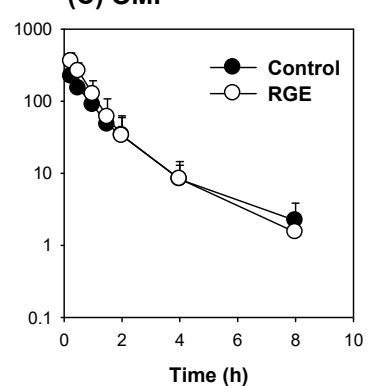

(G) Furosemide

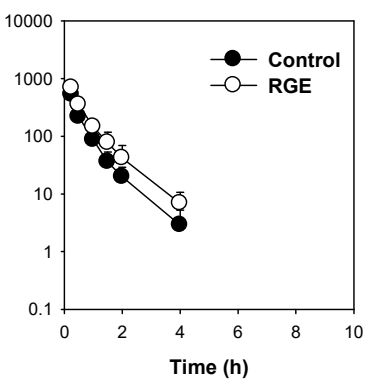

(D) NIF

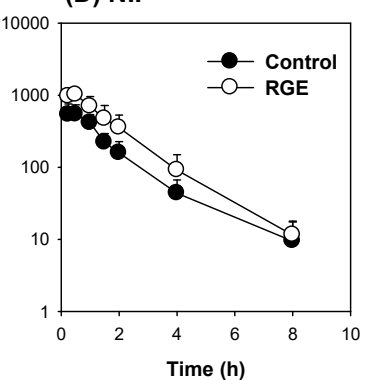

(H) Valsartan

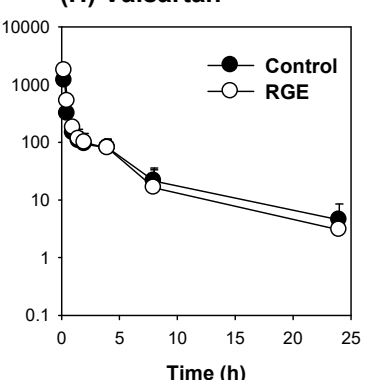

Figure 6. The plasma concentrations of (A) caffeine, (B) diclofenac (DCF), (C) omeprazole (OMP), (D) nifedipine (NIF), (E) dextromethorphan (DMX), (F) metformin, (G) furosemide, (H) valsartan, (I) digoxin, and (J) methotrexate following the repeated RGE administration ( $1.5 \mathrm{~g} / \mathrm{kg} / \mathrm{day}$ for 7 days) in rats that received dual cocktail sets. Dual cocktail set is composed of a mixture of CYP and transporter cocktails. The CYP cocktail consists of caffeine $(1 \mathrm{mg} / \mathrm{kg})$, diclofenac $(2 \mathrm{mg} / \mathrm{kg})$, omeprazole $(2 \mathrm{mg} / \mathrm{kg})$, nifedipine $(0.5 \mathrm{mg} / \mathrm{kg})$, and dextromethorphan $(10 \mathrm{mg} / \mathrm{kg})$ and orally administered. The transporter cocktail mixture is composed of metformin $(0.5 \mathrm{mg} / \mathrm{kg})$, furosemide $(0.1 \mathrm{mg} / \mathrm{kg})$, valsartan $(0.2 \mathrm{mg} / \mathrm{kg})$, digoxin $(2 \mathrm{mg} / \mathrm{kg})$, and methotrexate $(0.5 \mathrm{mg} / \mathrm{kg})$ and administered via intravenous injection. The data are presented as the means $\pm \mathrm{SD}(n=7)$. 

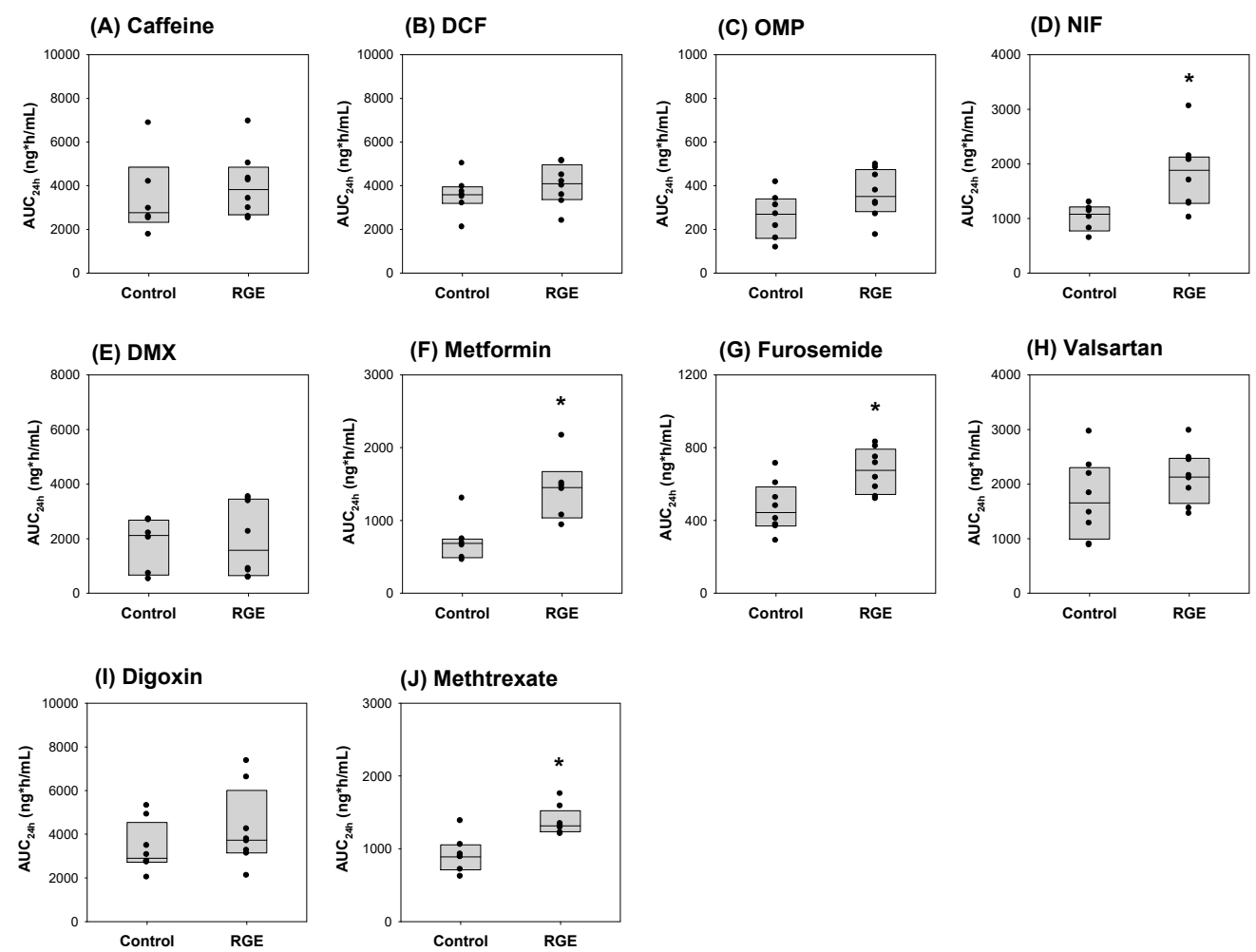

Figure 7. The comparison of AUC values of (A) caffeine, (B) diclofenac (DCF), (C) omeprazole (OMP), (D) nifedipine (NIF), (E) dextromethorphan (DMX), (F) metformin, (G) furosemide, (H) valsartan, (I) digoxin, and (J) methotrexate between the control and RGE treatment groups. ${ }^{*} p<0.05$ is considered statistically significant compared with the control group by the Mann-Whitney U test. The data are presented as the means $\pm \mathrm{SD}(n=7)$.

\section{Discussion}

With an increasing relevance of evaluating potential DDIs and HDIs, there is a need to increase the efficiency and speed of identifying these undesirable events. In the case of therapeutic drugs, in vitro to in vivo extrapolation and in silico PBPK modeling have been typically used for predicting DDIs [60]. Despite the increasing likelihood of HDIs, a standard in silico prediction system for evaluating HDIs remains elusive [61]. Currently, in vitro HDI evaluation in in vitro systems and preclinical animal study to estimate the inhibition and/or induction of drug-metabolizing enzymes and transporters are frequently used [61]. The separate investigation of potential CYP metabolizing enzymes and transporter mediated interactions can be a costly and time-consuming process. Therefore, several cocktail-based approaches to assess the potential in vitro and in vivo DDIs and HDIs have been developed [5,7-10]. Here, we developed a cocktail set composed of ten probe drugs for phenotyping both CYP metabolic enzymes and transporters in animal models simultaneously.

To develop the cocktail set, the simultaneous assessment of the probe substrates and their metabolites is critical. Here, we successfully developed the simultaneous determination method of 15 substrates and metabolites in the plasma with concentrations that ranged from $1 \mathrm{ng} / \mathrm{mL}(5 \mathrm{ng} / \mathrm{mL}$ for digoxin) to 1000 (for CYP substrates) or $2000 \mathrm{ng} / \mathrm{mL}$ (for transporter substrates). With the use of simultaneous analytical method of probe substrates and a series of well-designed pharmacokinetic studies, we demonstrated the lack of pharmacokinetic DDI among the 10 probe substrates (Tables 5-7).

The composition of the dual cocktail was determined according to the following probe drug selection criteria: (i) major CYP metabolizing enzymes and transporters in both human and rat; (ii) the safety and ease of access of probe drugs; (iii) the selectivity of probe substrates to the CYP enzymes or transporters of interest. The CYP cocktail set included probes for Cyp1a2, Cyp2c11, Cyp2d2, and Cyp3a1, which were selected based on previous cocktail sets developed for human and rat studies [7-10] because 
the consistency of the probe substrates of the in vivo cocktail among human and rats are critical in estimating the potential in vivo DDIs based on animal data. The homology of CYP orthologues between human and rats are approximately 70-75\% for CYP1A2 (Cyp1a2), CYP2C9 (Cyp2c11), CYP2D6 (Cyp2d2), CYP3A4 (Cyp3a1), although largely unknown for CYP2C19 (Cyp2c11) [62-64]. Cocktails designed with high consistency have great values in phenotyping metabolic enzyme and transporter activities in rats as models for different disease states [8,10]. They can be used to assess potential pharmacokinetic HDIs for herbal supplements that might be concomitantly administered with therapeutic drugs. The five probes CYP substrates, which included caffeine (Cyp1a2 in rat and CYP1A2 in human, $1 \mathrm{mg} / \mathrm{kg}$ ), diclofenac (Cyp2c11 in rat and CYP2C9 in human, $2 \mathrm{mg} / \mathrm{kg}$ ), omeprazole (Cyp2c11 in rat and CYP2C19 in human, $2 \mathrm{mg} / \mathrm{kg}$ ), dextromethorphan (Cyp2d2 in rat and CYP2D6 in human, $10 \mathrm{mg} / \mathrm{kg}$ ), nifedipine (Cyp3a1 in rat and CYP3A4 in human, $0.5 \mathrm{mg} / \mathrm{kg}$ ), were selected based on their use in other cocktails [7,18]. CYP2D6 is responsible for the metabolism of dextromethorphan to its major metabolite, dextrorphan. Dextromethorphan is also metabolized to 3-methoxymorphinan by CYP3A4 and subsequently metabolized to 3-hydroxymorphinan by CYP2D6. The formation of 3-methoxymorphinan by CYP3A4 is 2-17\% of dextrorphan formation [57]. A more than 200-fold decrease in dextrorphan formation was observed by the CYP2D6 compared with CYP2D6 poor metabolizer, suggesting the major contribution of CYP2D6 in the dextromethorphan metabolism [57]. In our study, no significant alterations in the plasma concentration of dextromethorphan by the ketoconazole treatment indicated the minor contribution of Сyp3a in the dextromethorphan pharmacokinetics. However, the involvement of multiple enzymes such as Сyp3a and Cyp2d and subsequent metabolic pathway from dextrorphan to hydroxymorphinan could be a confounding factor in pharmacokinetic DDIs or HDIs. Similar case should also be considered in omeprazole metabolism: Cyp3a-mediated omeprazole sulfone formation and Cyp2c-mediated 5-hydroxyomeprazole formation [56].

Meanwhile, we developed a dual cocktail that combines both CYP and transporter substrates owing to limited studies of substrate cocktails for drug transporter studies. The transporter cocktail set included probes for Oct1/2, Oat1/3, Oatp2, P-gp, and Mrp2, which play significant roles in the distribution and elimination of substrate drugs in the livers and kidneys [11]. Furthermore, these transporters shared similar substrate specificity among different species $[65,66]$. For example, an evaluation of 3300 Pfizer compounds in human P-gp showed fewer differences in substrate susceptibility than rodent P-gp [67], and the functional orthologues of human OCTs, OATs, OATPs, P-gp, and MRPs were identified in mice and rats [65]. The transporter substrate cocktail set included metformin (Oct1/2 in rat and OCT1/2 in human, $0.5 \mathrm{mg} / \mathrm{kg}$ ), furosemide (Oat1/3 in rat and OAT1/3 in human, $0.1 \mathrm{mg} / \mathrm{kg}$ ), valsartan (Oatp2 in rat and OATP1B1/1B3 in human, $0.2 \mathrm{mg} / \mathrm{kg}$ ), digoxin (P-gp in rat and human, $2 \mathrm{mg} / \mathrm{kg}$ ), and methotrexate (Mrp2 in rat and MRP2 in human, $0.5 \mathrm{mg} / \mathrm{kg}$ ) as probe substrates. These substrates were intravenously administered due to their low bioavailability, which is below 30\% in rats [13,22-26]. Their renal excretion was assessed since transporters, such as Oct, Oat, P-gp, and Mrp play significant roles in their substrate drug excretion $[11,13,20,21]$. Therefore, the transporter cocktail set could be used for the determination of pharmacokinetic alteration and disposition of probe substrates by the co-administered drugs or herbal supplements. However, we should note that transporter mediated DDIs and HDIs occurring in the intestinal absorption could not be detected in our dual cocktail system because of the intravenous injection of transporter cocktail set. Therefore, if the perpetrator is likely to interact with intestinal transporters such as P-gp, oral administrations of probe substrate such as digoxin concomitantly with perpetrator should be considered to investigate the intestinal DDIs or HDIs.

In addition, the dual cocktail set is designed to determine the pharmacokinetic DDIs or HDI related to the five CYP and five transporter activity modulation in rats; therefore, the investigation of pharmacodynamic interactions that might occur among the probe substrates or between probe substrate and perpetrator drug or herbal supplement could not be identified by the single low-dose administration of dual cocktail probe substrates.

An increased renal toxicity and a decreased renal excretion of methotrexate by the co-medication of nonsteroidal anti-inflammatory drugs, such as diclofenac, in the treatment of rheumatoid arthritis 
has been reported [68]. As the underlying mechanism, diclofenac-mediated OAT3 inhibition has been proposed with the $\mathrm{IC}_{50}$ values of diclofenac of $6.13 \mu \mathrm{M}$ [69]. However, Oat inhibition by diclofenac is unlikely in our cocktail set because the unbound maximum plasma concentration of diclofenac was calculated as $0.06 \mu \mathrm{M}$. The DDIs in hypertensive patients who were prescribed with antihypertensive, cardiovascular, and antidiabetic drugs are prevalent, and more than $55 \%$ and $32 \%$ of these DDIs were pharmacodynamic synergisms or antagonisms and increased serum potassium levels [70]. In our cocktail set, some antihypertensive drugs, such as nifedipine, furosemide, valsartan, and co-medicated antidiabetic and cardiovascular drugs, such as metformin and digoxin, could have possibility of these pharmacodynamic DDIs or altered potassium levels. However, as mentioned earlier, these pharmacodynamic DDIs or altered potassium levels is challenging to detect from the single administration of our dual cocktail set.

We also should note that large variations in the plasma concentrations of probe substrates, such as caffeine, omeprazole, nifedipine, dextromethorphan, metformin, and digoxin, at approximately 4-8 $\mathrm{h}$ that also resulted in the large variations in the AUC values of these substrates were observed during the development and validation stage of the dual cocktail set. Large variations in the urinal excretion $(\mathrm{Xu})$ were also observed in metformin and digoxin. These results are attributed to the difference in the elimination rate of individual rats. That is, two or three out of six rats showed fast elimination compared with other rats in our study group (Tables 5-7).

In the validation stage, a single oral dose of rifampin increased the plasma concentration of valsartan, which is due to the OATP inhibition by rifampin, and is consistent with the previous reports [21]; however, the plasma concentrations of other substrates were not changed by the single oral rifampin administration (Table 6). Multiple rifampin administration is known for inducing PXR and, consequently, inducing the CYP3A4 and P-gp transcription [54,55,71]. To avoid the direct inhibition by rifampin, the dual cocktail was administered $24 \mathrm{~h}$ after the last rifampin administration, according to the previous reports [42]. As expected, multiple rifampin treatment decreased the plasma concentrations of nifedipine and digoxin, which is caused by the increased CYP3A4 and P-gp activity. A single oral dose of ketoconazole increased the plasma concentrations of nifedipine and omeprazole. Based on the inhibitory effect of ketoconazole on CYP3a [72], a decreased dehydronifedipine formation and an increased nifedipine concentration in rat plasma could be expected, which was consistent with previous reports $[17,18]$. In case of omeprazole, CYP3A and CYP2C19 are involved in the omeprazole metabolism resulting in the formation of omeprazole sulfone and 5-hydroxyomeprazole, respectively [56]. Therefore, the ketoconazole treatment might inhibit the CYP3A4-mediated omeprazole metabolism and increase the plasma concentration of omeprazole; however, could not alter the formation of 5-hydroxyomeprazole (Table 6).

The concomitant administration of omeprazole decreased the itraconazole plasma concentration by decreasing its $\mathrm{pH}$-dependent dissolution in human [73], although did not significantly alter the plasma concentrations of itraconazole when orally administered [74]. It suggests the interaction between omeprazole and azole antifungal agent during the dissolution and absorption phase. In our study, we orally administered ketoconazole and administered omeprazole $1 \mathrm{~h}$ later at a dose of $2 \mathrm{mg} / \mathrm{kg}$ as the cocktail set. Considering that the $\mathrm{T}_{\max }$ of ketoconazole is $0.5-1 \mathrm{~h}$ in rats [75] and the effective omeprazole dose to increase gastric $\mathrm{pH}$ in rat is $20 \mathrm{mg} / \mathrm{kg}$ [76], the interaction between ketoconazole, and omeprazole during the absorption phase could be disregarded in this study.

Ketoconazole inhibited P-gp and CYP3A4 in both human and rats [77,78]. In humans, the in vivo $\mathrm{K}_{\mathrm{i}}$ value of ketoconazole for hepatic CYP3A4 and renal P-gp was estimated to be 6.64 and $2.27 \mathrm{ng} / \mathrm{mL}$, respectively [77]. In rats, the oral administration of ketoconazole at a dose of $50 \mathrm{mg} / \mathrm{kg}$ significantly increased the plasma concentrations of TAK-427, a P-gp substrate drug, following the oral and intravenous administration of TAK-427 [78]. However, in our case, we orally administered ketoconazole at a dose of $10 \mathrm{mg} / \mathrm{kg}$ in rats, which did not cause a significant alteration in the pharmacokinetics of digoxin, a probe substrate for P-gp (Table 6). This difference could be explained by the different dose of ketoconazole (50 mg/kg in the study of Takeuchi et al. [78] and $10 \mathrm{mg} / \mathrm{kg}$ in this study). Collectively, 
these results demonstrate the selectivity of known inhibitors, such as ketoconazole and rifampin, and known inducer rifampin in the assessment of pharmacokinetic DDIs of the dual cocktail set in rats. In addition, we used the novel dual cocktail set to detect the pharmacokinetic herb-drug interactions of RGE, one of the most popular herbal supplements. RGE pretreatment increased the plasma concentration of metformin, which is a specific probe for the Oct transporter. We previously demonstrated the tissue-specific regulation of Oct1 transporters in the intestine, liver, and kidneys [12]. The increased Oct1 expression in the enterocytes and decreased hepatic Oct1 expression coordinately increased the plasma concentration of metformin [12]. In this study, since metformin was intravenously administered, the increased plasma concentration of metformin could be due to the decreased hepatic Oct1 function. Similarly, Shu et al. [79] reported that the decreased OCT1 expression and function increased the plasma concentration of metformin in human. We also previously demonstrated the decreased Mrp2 expression in the rat liver following the repeated RGE treatment $(1.5 \mathrm{~g} / \mathrm{kg} / \mathrm{day}$ for seven days) and, consequently, the biliary excretion of methotrexate was decreased leading to an increased methotrexate plasma concentration [13], which is consistent with the observations in this study (Figure 7J). In addition, the plasma exposure of nifedipine and furosemide was also increased following the repeated RGE administration, which indicates the Cyp3a and OATS inhibition by RGE. The underlying mechanism(s) on the expression and function of Cyp3a and OATS by the multiple RGE exposure need to be further investigated. Taken together, the results demonstrate the applicability of the dual cocktail in effectively evaluating the pharmacokinetic herb-drug or drug-drug interactions by the simultaneous phenotyping of the activities of five CYP enzymes (Cyp1a, 2c, C9, 2C19, 2D6, and 3A4) and five drug transporters (OCTs, OATs, OATPs, P-gp, and MRP2).

In conclusion, we developed and validated a dual cocktail set, which is composed of 10 probe substrates, including caffeine (Cyp1a2 in rat and CYP1A2 in human, $1 \mathrm{mg} / \mathrm{kg}$ ), diclofenac (Cyp2c11 in rat and CYP2C9 in human, $2 \mathrm{mg} / \mathrm{kg}$ ), omeprazole (Cyp2c11 in rat and CYP2C19 in human, $2 \mathrm{mg} / \mathrm{kg}$ ), dextromethorphan (Cyp2d2 in rat and CYP2D6 in human, $10 \mathrm{mg} / \mathrm{kg})$, nifedipine (Cyp3a1 in rat and CYP3A4 in human, $0.5 \mathrm{mg} / \mathrm{kg}$ ), metformin (Oct1/2 in rat and OCT1/2 in human, $0.5 \mathrm{mg} / \mathrm{kg}$ ), furosemide (Oat1/3 in rat and OAT1/3 in human, $0.1 \mathrm{mg} / \mathrm{kg}$ ), valsartan (Oatp2 in rat and OATP1B1/1B3 in human, $0.2 \mathrm{mg} / \mathrm{kg}$ ), digoxin (P-gp in rat and human, $2 \mathrm{mg} / \mathrm{kg}$ ), and methotrexate (Mrp2 in rat and MRP2 in human, $0.5 \mathrm{mg} / \mathrm{kg}$ ). This dual cocktail set can be used to efficiently and simultaneously characterize the activity of five CYP enzymes and five transporters and effectively evaluate the pharmacokinetic drug-drug and herb-drug interactions through the modulation of five CYP enzymes and five transporters in rats.

Author Contributions: Conceptualization: I.-S.S.; methodology, M.K., J.-H.J., and M.-K.C.; validation, M.K., J.-H.J., and M.-K.C.; formal analysis, M.K.; investigation, M.K., J.-H.J., and M.-K.C.; writing-original draft preparation, M.K.; writing-review and editing, M.-K.C. and I.-S.S.; supervision, I.-S.S.; project administration, I.-S.S.; funding acquisition, I.-S.S. All authors have read and agreed to the published version of the manuscript.

Funding: This work was supported by a grant from the Korea Institute of Planning and Evaluation for Technology in Food, Agriculture, Forestry and Fisheries (IPET) through Export Promotion Technology Development Program, funded by the Ministry of Agriculture, Food and Rural Affairs (MAFRA) (No 316017-3), Republic of Korea and by the National Research Foundation of Korea (NRF) grant funded by the Korea government (MSIT) (No. NRF-2020R1A5A2017323 and NRF-2020R1I1A3074384).

Conflicts of Interest: The authors declare no conflict of interest.

\section{References}

1. Choi, M.K.; Song, I.S. Interactions of ginseng with therapeutic drugs. Arch. Pharmacal Res. 2019, 42, 862-878. [CrossRef] [PubMed]

2. Wu, X.; Ma, J.; Ye, Y.; Lin, G. Transporter modulation by Chinese herbal medicines and its mediated pharmacokinetic herb-drug interactions. J. Chromatogr. B Anal. Technol. Biomed. Life Sci. 2016, 1026, $236-253$. [CrossRef] [PubMed]

3. Adiwidjaja, J.; Boddy, A.V.; McLachlan, A.J. Physiologically based pharmacokinetic modelling of hyperforin to predict drug interactions with St John's wort. Clin. Pharmacokinet. 2019, 58, 911-926. [CrossRef] 
4. Brantley, S.; Graf, T.N.; Oberlies, N.H.; Paine, M.F. Physiologically-based pharmacokinetic interaction model framework for quantitative prediction of herb-drug interactions. Drug Metab. Rev. 2012, 44, 99-100.

5. Bosilkovska, M.; Samer, C.F.; Deglon, J.; Rebsamen, M.; Staub, C.; Dayer, P.; Walder, B.; Desmeules, J.A.; Daali, Y. Geneva cocktail for Cytochrome P450 and P-glycoprotein activity assessment using dried blood spots. Clin. Pharmacol. Ther. 2014, 96, 349-359. [CrossRef] [PubMed]

6. Derungs, A.; Donzelli, M.; Berger, B.; Noppen, C.; Krahenbuhl, S.; Haschke, M. Effects of Cytochrome P450 inhibition and induction on the phenotyping metrics of the Basel Cocktail: A randomized crossover study. Clin. Pharmacokinet. 2016, 55, 79-91. [CrossRef]

7. Ryu, J.Y.; Song, I.S.; Sunwoo, Y.E.; Shon, J.H.; Liu, K.H.; Cha, I.J.; Shin, J.G. Development of the "Inje cocktail” for high-throughput evaluation of five human cytochrome p450 Isoforms in vivo. Clin. Pharmacol. Ther. 2007, 82, 531-540. [CrossRef]

8. Uchida, S.; Tanaka, S.; Namiki, N. Simultaneous and comprehensive in vivo analysis of cytochrome P450 activity by using a cocktail approach in rats. Biopharm. Drug Dispos. 2014, 35, 228-236. [CrossRef]

9. Videau, O.; Delaforge, M.; Levi, M.; Thevenot, E.; Gal, O.; Becquemont, L.; Beaune, P.; Benech, H. Biochemical and analytical development of the CIME cocktail for drug fate assessment in humans. Rapid Commun. Mass Spectrom. 2010, 24, 2407-2419. [CrossRef]

10. Videau, O.; Pitarque, S.; Troncale, S.; Hery, P.; Thevenot, E.; Delaforge, M.; Benech, H. Can a cocktail designed for phenotyping pharmacokinetics and metabolism enzymes in human be used efficiently in rat? Xenobiotica 2012, 42, 349-354. [CrossRef]

11. International Transporter Consortium; Giacomini, K.M.; Huang, S.M.; Tweedie, D.J.; Benet, L.Z.; Brouwer, K.L.; Chu, X.; Dahlin, A.; Evers, R.; Fischer, V.; et al. Membrane transporters in drug development. Nat. Rev. Drug Discov. 2010, 9, 215-236. [CrossRef] [PubMed]

12. Sotaniemi, E.A.; Haapakoski, E.; Rautio, A. Ginseng therapy in non-insulin-dependent diabetic patients. Diabetes Care 1995, 18, 1373-1375. [CrossRef] [PubMed]

13. Lee, S.; Kwon, M.; Choi, M.K.; Song, I.S. Effects of red ginseng extract on the pharmacokinetics and elimination of methotrexate via Mrp2 regulation. Molecules 2018, 23, 2948. [CrossRef] [PubMed]

14. FDA. In Vitro Drug Interaction Studies-Cytochrome P450 Enzyme- and Transporter-Mediated Drug Interactions Guidance for Industry. Available online: https:/www.fda.gov/regulatory-information/searchfda-guidance-documents/clinical-drug-interaction-studies-cytochrome-p450-enzyme-and-transportermediated-drug-interactions (accessed on 30 September 2020).

15. EMA. Guideline on the investigation of drug interactions. React. Wkly. 2012. [CrossRef]

16. Liska, D.J. The detoxification enzyme systems. Altern. Med. Rev. 1998, 3, 187-198.

17. Choi, J.S.; Choi, I.; Choi, D.H. Effects of pioglitazone on the pharmacokinetics of nifedipine and its main metabolite, dehydronifedipine, in rats. Eur. J. Drug Metab. Pharmacokinet. 2016, 41, 231-238. [CrossRef]

18. Choi, Y.H.; Lee, M.G. Pharmacokinetic and pharmacodynamic interaction between nifedipine and metformin in rats: Competitive inhibition for metabolism of nifedipine and metformin by each other via CYP isozymes. Xenobiotica 2012, 42, 483-495. [CrossRef]

19. Chovan, J.P.; Ring, S.C.; Yu, E.; Baldino, J.P. Cytochrome P450 probe substrate metabolism kinetics in Sprague Dawley rats. Xenobiotica 2007, 37, 459-473. [CrossRef]

20. Yamashiro, W.; Maeda, K.; Hirouchi, M.; Adachi, Y.; Hu, Z.; Sugiyama, Y. Involvement of transporters in the hepatic uptake and biliary excretion of valsartan, a selective antagonist of the angiotensin II AT1-receptor, in humans. Drug Metab. Dispos. 2006, 34, 1247-1254. [CrossRef]

21. Dong, H.; Ma, J.; Li, T.; Xiao, Y.; Zhang, N.; Liu, J.; Gao, Y.; Shao, J.; Jia, L. Global deregulation of ginseng products may be a safety hazard to warfarin takers: Solid evidence of ginseng-warfarin interaction. Sci. Rep. 2017, 7, 5813. [CrossRef]

22. Peng, S.X.; Ritchie, D.M.; Cousineau, M.; Danser, E.; Dewire, R.; Floden, J. Altered oral bioavailability and pharmacokinetics of P-glycoprotein substrates by coadministration of biochanin A. J. Pharmacol. Sci. 2006, 95, 1984-1993. [CrossRef] [PubMed]

23. Kim, E.J.; Han, K.S.; Lee, M.G. Gastrointestinal first-pass effect of furosemide in rats. J. Pharm. Pharmacol. 2000, 52, 1337-1343. [CrossRef] [PubMed]

24. Choi, Y.H.; Kim, S.G.; Lee, M.G. Dose-independent pharmacokinetics of metformin in rats: Hepatic and gastrointestinal first-pass effects. J. Pharmacol. Sci. 2006, 95, 2543-2552. [CrossRef] 
25. Lee, J.Y.; Kang, W.S.; Piao, J.; Yoon, I.S.; Kim, D.D.; Cho, H.J. Soluplus (R)/TPGS-based solid dispersions prepared by hot-melt extrusion equipped with twin-screw systems for enhancing oral bioavailability of valsartan. Drug Des. Dev. Ther. 2015, 9, 2745-2756.

26. Chen, C.P.; Scott, D.; Hanson, E.; Franco, J.; Berryman, E.; Volberg, M.; Liu, X.R. Impact of mrp2 on the biliary excretion and intestinal absorption of furosemide, probenecid, and methotrexate using Eisai hyperbilirubinemic rats. Pharm. Res. 2003, 20, 31-37. [CrossRef] [PubMed]

27. Lee, C.H.; Kim, J.H. A review on the medicinal potentials of ginseng and ginsenosides on cardiovascular diseases. J. Ginseng Res. 2014, 38, 161-166. [CrossRef] [PubMed]

28. Ru, W.; Wang, D.; Xu, Y.; He, X.; Sun, Y.E.; Qian, L.; Zhou, X.; Qin, Y. Chemical constituents and bioactivities of Panax ginseng (C. A. Mey.). Drug Discov. Ther. 2015, 9, 23-32. [CrossRef]

29. Gurley, B.J.; Gardner, S.F.; Hubbard, M.A.; Williams, D.K.; Gentry, W.B.; Cui, Y.; Ang, C.Y. Clinical assessment of effects of botanical supplementation on cytochrome P450 phenotypes in the elderly: St John's wort, garlic oil, Panax ginseng and Ginkgo biloba. Drugs Aging 2005, 22, 525-539. [CrossRef]

30. Gurley, B.J.; Gardner, S.F.; Hubbard, M.A.; Williams, D.K.; Gentry, W.B.; Cui, Y.; Ang, C.Y. Cytochrome P450 phenotypic ratios for predicting herb-drug interactions in humans. Clin. Pharmacol. Ther. 2002, 72, $276-287$. [CrossRef]

31. Kim, D.S.; Kim, Y.; Jeon, J.Y.; Kim, M.G. Effect of red ginseng on cytochrome P450 and P-glycoprotein activities in healthy volunteers. J. Ginseng Res. 2016, 40, 375-381. [CrossRef]

32. Seong, S.J.; Kang, W.Y.; Heo, J.K.; Jo, J.; Choi, W.G.; Liu, K.H.; Lee, S.; Choi, M.K.; Han, Y.H.; Lee, H.S.; et al. A comprehensive in vivo and in vitro assessment of the drug interaction potential of red ginseng. Clin. Ther. 2018, 40, 1322-1337. [CrossRef] [PubMed]

33. Malati, C.Y.; Robertson, S.M.; Hunt, J.D.; Chairez, C.; Alfaro, R.M.; Kovacs, J.A.; Penzak, S.R. Influence of panax ginseng on cytochrome P450 (CYP)3A and P-glycoprotein (P-gp) activity in healthy participants. J. Clin. Pharmacol. 2012, 52, 932-939. [CrossRef] [PubMed]

34. Kim, H.; Nam, W.; Kim, S.H.; Jang, H.R.; Lee, M.K.; Kim, T.W.; Lee, S. Modulatory effects of Korean red ginseng extract (Panax ginseng C.A. Meyer) on cytochrome P450 after oral administration to mice for 14 days. J. Life Sci. 2012, 22, 991-998. [CrossRef]

35. Bogacz, A.; Karasiewicz, M.; Dziekan, K.; Procyk, D.; Górska-Paukszta, M.; Kowalska, A.; Mikolajczak, P.L.; Ożarowski, M.; Czerny, B. Impact of Panax ginseng and Ginkgo biloba extracts on expression level of transcriptional factors and xenobiotic-metabolizing cytochrome P450 enzymes. Herba Pol. 2016, 62, $42-54$. [CrossRef]

36. Hong, B.N.; Ji, M.G.; Kang, T.H. The efficacy of red ginseng in type 1 and type 2 diabetes in animals. Evid. Based Complement. Altern. Med. 2013, 2013, 593181. [CrossRef] [PubMed]

37. Hong, Y.J.; Kim, N.; Lee, K.; Hee Sonn, C.; Eun Lee, J.; Tae Kim, S.; Ho Baeg, I.; Lee, K.M. Korean red ginseng (Panax ginseng) ameliorates type 1 diabetes and restores immune cell compartments. J. Ethnopharmacol. 2012, 144, 225-233. [CrossRef] [PubMed]

38. Hyun, H.B.; Lee, W.S.; Go, S.I.; Nagappan, A.; Park, C.; Han, M.H.; Hong, S.H.; Kim, G.; Kim, G.Y.; Cheong, J.; et al. The flavonoid morin from Moraceae induces apoptosis by modulation of Bcl-2 family members and Fas receptor in HCT 116 cells. Int. J. Oncol. 2015, 46, 2670-2678. [CrossRef]

39. Nam, S.J.; Han, Y.J.; Lee, W.; Kang, B.; Choi, M.K.; Han, Y.H.; Song, I.S. Effect of red ginseng extract on the pharmacokinetics and efficacy of metformin in streptozotocin-induced diabetic rats. Pharmaceutics 2018, 10, 80. [CrossRef]

40. Chen, S.; Wang, Z.; Huang, Y.; O’Barr, S.A.; Wong, R.A.; Yeung, S.; Chow, M.S. Ginseng and anticancer drug combination to improve cancer chemotherapy: A critical review. Evid. Based Complement. Alternat. Med. 2014, 2014, 168940. [CrossRef]

41. Endale, M.; Im, E.J.; Lee, J.Y.; Kim, S.D.; Yayeh, T.; Song, Y.B.; Kwak, Y.S.; Kim, C.; Kim, S.H.; Roh, S.S.; et al. Korean red ginseng saponin fraction rich in ginsenoside-Rb1, Rc and Rb2 attenuates the severity of mouse collagen-induced arthritis. Mediat. Inflamm. 2014, 2014, 748964. [CrossRef]

42. Zheng, H.X.; Huang, Y.; Frassetto, L.A.; Benet, L.Z. Elucidating rifampin's inducing and inhibiting effects on glyburide pharmacokinetics and blood glucose in healthy volunteers: Unmasking the differential effects of enzyme induction and transporter inhibition for a drug and its primary metabolite. Clin. Pharmacol. Ther. 2009, 85, 78-85. [CrossRef] [PubMed] 
43. Heo, J.K.; Kim, H.J.; Lee, G.H.; Ohk, B.; Lee, S.; Song, K.S.; Song, I.S.; Liu, K.H.; Yoon, Y.R. Simultaneous determination of five Cytochrome $\mathrm{P} 450$ probe substrates and their metabolites and organic anion transporting polypeptide probe substrate in human plasma using liquid chromatography-tandem mass spectrometry. Pharmaceutics 2018, 10, 79. [CrossRef] [PubMed]

44. Jin, S.; Lee, S.; Jeon, J.H.; Kim, H.; Choi, M.K.; Song, I.S. Enhanced intestinal permeability and plasma concentration of metformin in rats by the repeated administration of red ginseng extract. Pharmaceutics 2019, 11, 189. [CrossRef] [PubMed]

45. Jeon, J.H.; Lee, S.; Lee, W.; Jin, S.; Kwon, M.; Shin, C.H.; Choi, M.K.; Song, I.S. Herb-drug interaction of red ginseng extract and ginsenoside Rc with valsartan in rats. Molecules 2020, 25, 622. [CrossRef]

46. Frommherz, L.; Kohler, H.; Brinkmann, B.; Lehr, M.; Beike, J. LC-MS assay for quantitative determination of cardio glycoside in human blood samples. Int. J. Leg. Med. 2008, 122, 109-114. [CrossRef]

47. Keane, S.; Wallace, G.; Munday, C.; Wright, M. Sensitive and robust LC-MS/MS analysis of digoxin in human plasma through optimization of in-source adduct formation. Bioanalysis 2018, 10, 1401-1411. [CrossRef]

48. Kim, S.; Choi, W.G.; Kwon, M.; Lee, S.; Cho, Y.Y.; Lee, J.Y.; Kang, H.C.; Song, I.S.; Lee, H.S. In vitro inhibitory effects of APINACA on human major Cytochrome P450, UDP-Glucuronosyltransferase enzymes, and drug transporters. Molecules 2019, 24, 3000. [CrossRef]

49. Song, I.S.; Kong, T.Y.; Jeong, H.U.; Kim, E.N.; Kwon, S.S.; Kang, H.E.; Choi, S.Z.; Son, M.; Lee, H.S. Evaluation of the transporter-mediated herb-drug interaction potential of DA-9801, a standardized dioscorea extract for diabetic neuropathy, in human in vitro and rat in vivo. BMC Complement. Altern. Med. 2014, 14, e251. [CrossRef]

50. Zimmer, D. New US FDA draft guidance on bioanalytical method validation versus current FDA and EMA guidelines: Chromatographic methods and ISR. Bioanalysis 2014, 6, 13-19. [CrossRef]

51. Wang, J.S.; Wen, X.; Backman, J.T.; Taavitsainen, P.; Neuvonen, P.J.; Kivisto, K.T. Midazolam alpha-hydroxylation by human liver microsomes in vitro: Inhibition by calcium channel blockers, itraconazole and ketoconazole. Pharmacol. Toxicol. 1999, 85, 157-161. [CrossRef]

52. Lee, K.S.; Park, J.H.; Lee, H.J.; Rhie, S. Preclinical study of a potent P-glycoprotein and cytochrome P450 enzyme inducer rifampicin changing pharmacokinetic parameters of risperidone and its metabolite, 9-hydroxyrisperidone, using a rat model. J. Pharm. Investig. 2012, 42, 345-351. [CrossRef]

53. Yigitaslan, S.; Erol, K.; Cengelli, C. The effect of P-glycoprotein Inhibition and activation on the absorption and serum levels of cyclosporine and tacrolimus in rats. Adv. Clin. Exp. Med. 2016, 25, 237-242. [CrossRef] [PubMed]

54. Chang, J.H.; Ly, J.; Plise, E.; Zhang, X.; Messick, K.; Wright, M.; Cheong, J. Differential effects of rifampin and ketoconazole on the blood and liver concentration of atorvastatin in wild-type and Cyp3a and Oatp1a/b knockout mice. Drug. Metab. Dispos. 2014, 42, 1067-1073. [CrossRef] [PubMed]

55. Tanaka, C.; Yin, O.Q.; Smith, T.; Sethuraman, V.; Grouss, K.; Galitz, L.; Harrell, R.; Schran, H. Effects of rifampin and ketoconazole on the pharmacokinetics of nilotinib in healthy participants. J. Clin. Pharmacol. 2011, 51, 75-83. [CrossRef] [PubMed]

56. Park, E.J.; Cho, H.Y.; Lee, Y.B. Effect of cimetidine and phenobarbital on metabolite kinetics of omeprazole in rats. Arch. Pharmacal Res. 2005, 28, 1196-1202. [CrossRef] [PubMed]

57. Takashima, T.; Murase, S.; Iwasaki, K.; Shimada, K. Evaluation of dextromethorphan metabolism using hepatocytes from CYP2D6 poor and extensive metabolizers. Drug Metab. Pharmacokinet. 2005, 20, 177-182. [CrossRef]

58. Masuda, M.; I'Izuka, Y.; Yamazaki, M.; Nishigaki, R.; Kato, Y.; Ni'inuma, K.; Suzuki, H.; Sugiyama, Y. Methotrexate is excreted into the bile by canalicular multispecific organic anion transporter in rats. Cancer Res. 1997, 57, 3506-3510.

59. Poirier, A.; Cascais, A.C.; Funk, C.; Lave, T. Prediction of pharmacokinetic profile of valsartan in human based on in vitro uptake transport data. J. Pharmacokinet. Pharmacodyn. 2009, 36, 585-611. [CrossRef]

60. Sager, J.E.; Yu, J.; Ragueneau-Majlessi, I.; Isoherranen, N. Physiologically based pharmacokinetic (PBPK) modeling and simulation approaches: A systematic review of published models, applications, and model verification. Drug Metab. Dispos. 2015, 43, 1823-1837. [CrossRef]

61. Brantley, S.J.; Argikar, A.A.; Lin, Y.S.; Nagar, S.; Paine, M.F. Herb-drug interactions: Challenges and opportunities for improved predictions. Drug Metab. Dispos. 2014, 42, 301-317. [CrossRef]

62. Lewis, D.F. Homology modelling of human cytochromes P450 involved in xenobiotic metabolism and rationalization of substrate selectivity. Exp. Toxicol. Pathol. 1999, 51, 369-374. [CrossRef] 
63. Sakai, C.; Iwano, S.; Yamazaki, Y. Species Differences in the Pharmacokinetic Parameters of Cytochrome P450 Probe Substrates between Experimental Animals, such as Mice, Rats, Dogs, Monkeys, and Microminipigs, and Humans. J. Drug Metab. Toxicol. 2014, 5, 1000173.

64. Martignoni, M.; Groothuis, G.M.M.; de Kanter, R. Species differences between mouse, rat, dog, monkey and human CYP-mediated drug metabolism, inhibition and induction. Expert Opin. Drug Metab. Toxicol. 2006, 2, 875-894. [CrossRef] [PubMed]

65. Chu, X.Y.; Bleasby, K.; Evers, R. Species differences in drug transporters and implications for translating preclinical findings to humans. Expert Opin. Drug Metab. Toxicol. 2013, 9, 237-252. [CrossRef]

66. Choi, M.K.; Song, I.S. Organic Cation Transporters and their Pharmacokinetic and Pharmacodynamic Consequences. Drug Metab. Pharmacokinet. 2008, 23, 243-253. [CrossRef]

67. Feng, B.; Mills, J.B.; Davidson, R.E.; Mireles, R.J.; Janiszewski, J.S.; Troutman, M.D.; de Morais, S.M. In vitro P-glycoprotein assays to predict the in vivo interactions of P-glycoprotein with drugs in the central nervous system. Drug Metab. Dispos. 2008, 36, 268-275. [CrossRef]

68. Svanstrom, H.; Lund, M.; Melbye, M.; Pasternak, B. Concomitant use of low-dose methotrexate and NSAIDs and the risk of serious adverse events among patients with rheumatoid arthritis. Pharmacoepidemiol. Drug Saf. 2018, 27, 885-893. [CrossRef]

69. Ni, Y.; Duan, Z.L.; Zhou, D.D.; Liu, S.; Wan, H.D.; Gui, C.S.; Zhang, H.J. Identification of structural features for the inhibition of OAT3-mediated uptake of enalaprilat by selected drugs and flavonoids. Front. Pharmacol. 2020, 11, e802. [CrossRef]

70. Kothari, N.; Ganguly, B. Potential drug-drug interactions among medications prescribed to hypertensive patients. J. Clin. Diagn. Res. 2014, 8, HC01-HC04. [CrossRef]

71. Greiner, B.; Eichelbaum, M.; Fritz, P.; Kreichgauer, H.P.; Von Richter, O.; Zundler, J.; Kroemer, H.K. The role of intestinal P-glycoprotein in the interaction of digoxin and rifampin. J. Clin. Investig. 1999, 104, 147-153. [CrossRef]

72. Kimoto, E.; Vourvahis, M.; Scialis, R.J.; Eng, H.; Rodrigues, A.D.; Varma, M.V.S. Mechanistic evaluation of the complex drug-drug interactions of maraviroc: Contribution of Cytochrome P450 3A, P-glycoprotein and Organic Anion Transporting Polypeptide 1B1. Drug Metab. Dispos. 2019, 47, 493-503. [CrossRef] [PubMed]

73. Jaruratanasirikul, S.; Sriwiriyajan, S. Effect of omeprazole on the pharmacokinetics of itraconazole. Eur. J. Clin. Pharmacol. 1998, 54, 159-161. [CrossRef] [PubMed]

74. Johnson, M.D.; Hamilton, C.D.; Drew, R.H.; Sanders, L.L.; Pennick, G.J.; Perfect, J.R. A randomized comparative study to determine the effect of omeprazole on the peak serum concentration of itraconazole oral solution. J. Antimicrob. Chemother. 2003, 51, 453-457. [CrossRef] [PubMed]

75. Remmel, R.P.; Amoh, K.; Abdel-Monem, M.M. The disposition and pharmacokinetics of ketoconazole in the rat. Drug Metab. Dispos. 1987, 15, 735-739.

76. Segawa, K.; Nakazawa, S.; Tsukamoto, Y.; Chujoh, C.; Yamao, K.; Hase, S. Effect of omeprazole on gastric acid secretion in rat: Evaluation of dose, duration of effect, and route of administration. Gastroenterol. Jpn. 1987, 22, 413-418. [CrossRef]

77. Oishi, M.; Takano, Y.; Torita, Y.; Malhotra, B.; Chiba, K. Physiological based pharmacokinetic modeling to estimate in vivo Ki of ketoconazole on renal P-gp using human drug-drug interaction study result of fesoterodine and ketoconazole. Drug Metab. Pharmacokinet. 2018, 33, 90-95. [CrossRef]

78. Takeuchi, T.; Nonaka, M.; Yoshitomi, S.; Higuchi, T.; Ebihara, T.; Maeshiba, Y.; Kawase, M.; Asahi, S. Marked impact of P-glycoprotein on the absorption of TAK-427 in rats. Biopharm. Drug Dispos. 2008, 29, 311-323. [CrossRef]

79. Shu, Y.; Sheardown, S.A.; Brown, C.; Owen, R.P.; Zhang, S.; Castro, R.A.; Ianculescu, A.G.; Yue, L.; Lo, J.C.; Burchard, E.G.; et al. Effect of genetic variation in the organic cation transporter 1 (OCT1) on metformin action. J. Clin. Investig. 2007, 117, 1422-1431. [CrossRef]

(C) 2020 by the authors. Licensee MDPI, Basel, Switzerland. This article is an open access article distributed under the terms and conditions of the Creative Commons Attribution (CC BY) license (http://creativecommons.org/licenses/by/4.0/). 